\title{
Evidence for Long-Timescale Patterns of Synaptic Inputs in CA1 of Awake Behaving Mice
}

\author{
IDIlya Kolb, ${ }^{1 \star}$ Giovanni Talei Franzesi, ${ }^{2 \star}$ Michael Wang, ${ }^{1}$ SSuhasa B. Kodandaramaiah, ${ }^{3}$ CCraig R. Forest, ${ }^{4}$ \\ Edward S. Boyden, ${ }^{2,5}$ and Annabelle C. Singer ${ }^{1}$ \\ ${ }^{1}$ Wallace H. Coulter Department of Biomedical Engineering, Georgia Institute of Technology and Emory University, Atlanta, Georgia 30332, ${ }^{2}$ MIT Media \\ Lab, Massachusetts Institute of Technology, Cambridge, Massachusetts 02139, ${ }^{3}$ Department of Mechanical Engineering, University of Minnesota, \\ Minneapolis, Minnesota 55455, ${ }^{4}$ George W. Woodruff School of Mechanical Engineering, Georgia Institute of Technology, Atlanta, Georgia 30332, and \\ ${ }^{5} \mathrm{McGovern} \mathrm{Institute,} \mathrm{Massachusetts} \mathrm{Institute} \mathrm{of} \mathrm{Technology,} \mathrm{Cambridge,} \mathrm{Massachusetts} 02139$
}

Repeated sequences of neural activity are a pervasive feature of neural networks in vivo and in vitro. In the hippocampus, sequential firing of many neurons over periods of $100-300 \mathrm{~ms}$ reoccurs during behavior and during periods of quiescence. However, it is not known whether the hippocampus produces longer sequences of activity or whether such sequences are restricted to specific network states. Furthermore, whether long repeated patterns of activity are transmitted to single cells downstream is unclear. To answer these questions, we recorded intracellularly from hippocampal CA1 of awake, behaving male mice to examine both subthreshold activity and spiking output in single neurons. In eight of nine recordings, we discovered long $(900 \mathrm{~ms})$ reoccurring subthreshold fluctuations or "repeats." Repeats generally were high-amplitude, nonoscillatory events reoccurring with $10 \mathrm{~ms}$ precision. Using statistical controls, we determined that repeats occurred more often than would be expected from unstructured network activity (e.g., by chance). Most spikes occurred during a repeat, and when a repeat contained a spike, the spike reoccurred with precision on the order of $\leq 20 \mathrm{~ms}$, showing that long repeated patterns of subthreshold activity are strongly connected to spike output. Unexpectedly, we found that repeats occurred independently of classic hippocampal network states like theta oscillations or sharp-wave ripples. Together, these results reveal surprisingly long patterns of repeated activity in the hippocampal network that occur nonstochastically, are transmitted to single downstream neurons, and strongly shape their output. This suggests that the timescale of information transmission in the hippocampal network is much longer than previously thought.

Key words: hippocampus; intracellular activity; subthreshold patterns

Significance Statement

We found long ( $\geq 900 \mathrm{~ms}$ ), repeated, subthreshold patterns of activity in CA1 of awake, behaving mice. These repeated patterns ("repeats") occurred more often than expected by chance and with $10 \mathrm{~ms}$ precision. Most spikes occurred within repeats and reoccurred with a precision on the order of $20 \mathrm{~ms}$. Surprisingly, there was no correlation between repeat occurrence and classical network states such as theta oscillations and sharp-wave ripples. These results provide strong evidence that long patterns of activity are repeated and transmitted to downstream neurons, suggesting that the hippocampus can generate longer sequences of repeated activity than previously thought.

\section{Introduction}

Precise firing sequences may be a universal, emergent property of neural networks, having been observed in computational models of neural networks (Abeles, 1991; Diesmann et al., 1999; Fiete et al., 2010), neuronal cultures (Rolston et al., 2007), acute brain slices (MacLean et al., 2005), and in vivo (Shmiel et al., 2005; 
Luczak et al., 2009; Harvey et al., 2012; Miller et al., 2014; Carrillo-Reid et al., 2016). While sequential activity with fine temporal precision can represent information over time (Mauk and Buonomano, 2004), such precise sequences can also be internally generated, in the absence of an external stimulus (Luczak and Maclean, 2012). For example, in hippocampal CA3, sequential firing of neurons on the timescale of 100-300 ms (or up to $\sim 500 \mathrm{~ms}$ in extended replay; Davidson et al., 2009) reoccurs during behavior and during periods of quiescence (replay or reactivation, and preplay; Nádasdy et al., 1999; Louie and Wilson, 2001; Dragoi and Tonegawa, 2011), and is tied to sharp-wave ripples (SWRs), thought to play an important role in learning and memory (Girardeau et al., 2009; Diekelmann and Born, 2010; Carr et al., 2011; Jadhav et al., 2012).

Elucidating the duration of repeated sequences and how such sequences influence downstream activity is important for understanding how information is encoded. However, little attention has been paid to how these sequences are transmitted to neurons downstream. It remains unclear, for example, whether structured sequences of repeated spiking activity are transmitted to single neurons in CA1, in which case single neurons would have long repeated patterns of subthreshold activity, or whether each downstream neuron receives inputs only from short segments of the sequence. Long repeated intracellular patterns would provide evidence that information transmission to single cells can occur over long timescales. In turn, since subthreshold dynamics shape spiking output, the presence of long repeated intracellular patterns might suggest that information encoded over such long timescales is further relayed to downstream neurons. If that is indeed the case, we might expect the spiking output within repeated intracellular activity to also repeat with high accuracy, possibly on the order of a few milliseconds. Two basic questions are how long such repeated sequences last and whether they are specific to particular network states (e.g., SWRs) or they represent a more general feature of hippocampal information processing.

Prior work has examined repeated patterns of intracellular activity in vitro and in anesthetized animals (Ikegaya et al., 2004, 2008; Mokeichev et al., 2007); however, whether such repeated activity occurs in the awake brain is unknown. Ikegaya et al. (2004) previously recorded activity resembling brief spontaneous membrane voltage fluctuations, likely isolated postsynaptic inputs, superimposed on a low-variance stable baseline in acute brain slices. Similarly, in previous in vivo anesthetized studies (Ikegaya et al., 2004; Mokeichev et al., 2007) spontaneous intracellular activity tended to exhibit periodic subthreshold $\sim 1 \mathrm{~Hz}$ fluctuations ("up-down states"; Stern et al., 1997), which are associated with various forms of anesthesia (Contreras and Steriade, 1995; Li et al., 2009). Both of these activity patterns have a structure that is not present in awake animals. In neurons of awake, behaving animals, activity is less stereotyped due to the nonsynchronous activation of presynaptic populations, neuromodulator action, and changes in conductance state (Steriade et al., 2001; Haider et al., 2006; Constantinople and Bruno, 2011). Because of these differences in membrane voltage dynamics between anesthetized and awake animals, one cannot assume that the prevalence and properties of long intracellular repeats identified in anesthetized animals will also hold in awake animals.

Coulter Department of Biomedical Engineering, Georgia Institute of Technology, Atlanta, GA 30332, E-mail: asinger@gatech.edu.

DOI:10.1523/JNEUROSCI.1519-17.2017

Copyright $\odot 2018$ the authors $\quad 0270-6474 / 18 / 381822-14 \$ 15.00 / 0$
To address these questions, we recorded intracellular neural activity via patch-clamp recording from neurons in awake mice running on a spherical treadmill. Intracellular recordings are particularly suitable for this analysis, both because they remove the risk of spike misattribution and because the recorded neuron acts as a microphone, "listening" to all its synaptic patterns, which could not be recorded extracellularly at the given state of technology. Because repeated spiking patterns have been well characterized in CA3, we recorded from neurons in hippocampal CA1, which receives inputs from $\mathrm{CA} 3$ as well as entorhinal cortex and the nucleus reuniens of the thalamus. We searched for sequences of intracellular activity on timescales at which repeated spiking activity has previously been described (300 ms) as well as over longer timescales $(900 \mathrm{~ms})$, finding repeated subthreshold activity occurring at both of these timescales independently of network states. Importantly, such repeats occurred in a variety of network and behavioral states, suggesting that they are a basic feature of hippocampal information coding and transmission. To assess whether the subthreshold patterns occurred by chance, we compared incidences of repeated patterns in real data to simulated subthreshold activity and shuffled recordings (Mokeichev et al., 2007). We found there were more incidences of repeated activity in real data than in these statistical controls. However, prior studies have found fault with using only statistical control to establish whether or not repeats are stochastic. In previous studies, surrogate data generated using stochastic mechanisms exhibited approximately the same number of repeats as real physiological data, suggesting that repeats in membrane voltage were likely generated by random processes (Mokeichev et al., 2007). Similar conclusions have been reached when analyzing spiking activity (Oram et al., 1999; Roxin et al., 2008). On the other hand, recent evidence suggests that membrane potential repeat occurrence may be significant (nonrandom) in some preparations but not others, depending strongly on the specifics of the repeat detection method and the recording conditions (Ikegaya et al., 2008). Therefore, we additionally examined the precision of repeated intracellular patterns because the existence of millisecondtimescale precision in these repeats would contribute to evidence that these patterns are not random within the physiological time frame of neuronal information processing. We found that these subthreshold patterns were repeated with a precision of $\sim 10 \mathrm{~ms}$. Finally, we found that most spikes occur during repeated intracellular activity, providing evidence for a mechanism by which repeated neural activity patterns could propagate in this network: $82 \%$ of spikes occurred within a repeat and spikes within repeats reoccurred with high precision.

These results are the first evidence of repeated patterns of intracellular activity on these long timescales in the awake brain. We show that long patterns of activity are repeated and transmitted to downstream neurons, revealing a long timescale of information transmission. The methods used here could be applied to reveal timescales of information transmission in other brain circuits.

\section{Materials and Methods}

Surgical procedures, behavioral training, electrophysiology. A subset of the raw data used in this study was used in a previous publication (Singer et al., 2017); however, all the analyses presented here are new and independent.

All animal procedures were approved by the MIT Committee on Animal Care. For a detailed description of the experimental setup, refer to the study by Singer et al. (2017). Briefly, adult male C57BL/6 mice 8-12 weeks old were implanted with custom-made headplates. They were left to recover from the surgery for 1 week and habituated to handling for 1-2 
$\mathrm{d}$ before behavioral training began. Animals were trained to run on an 8 inch spherical treadmill, as described by Harvey et al. (2009) in a dimly lit room. The animals engaged in spontaneous behavior (e.g., running, grooming). Animals learned to run on the treadmill over $\sim 1$ week. To acclimate to the testing environment, on the first $2 \mathrm{~d}$ of training the animals were placed on the spherical treadmill and rewarded with undiluted sweetened condensed milk. From the third day until the end of training (typically 5-7 d), the animals were placed on the treadmill for increasing amounts of time ( $30 \mathrm{~min}$ to $2 \mathrm{~h}$ ).

On the day of the experiment, two craniotomies (diameter, 200-400 $\mu \mathrm{m}$ ) were performed at the following locations (in $\mathrm{mm}$ from bregma): 2 caudal, 1.4 lateral for whole-cell recording, and 3.23 anterior, and 0.58 lateral for local field potential (LFP) recording. Standard patch-clamp pipettes were pulled from filamented glass capillaries using a P97 Pipette Puller (Sutter Instruments) to a resistance of 4-9 M $\Omega$. The intracellular pipette solution consisted of the following (in $\mathrm{mm}$ ): 125 potassium gluconate, $0.1 \mathrm{CaCl} 2,0.6 \mathrm{MgCl} 2,1 \mathrm{EGTA}, 10$ HEPES, $4 \mathrm{Mg}$ ATP, $0.4 \mathrm{Na}$ $\mathrm{GTP}$, and $8 \mathrm{NaCl}$. For biocytin staining, $500 \mu \mathrm{M}$ biocytin, sodium salt (Invitrogen), was added to the pipette solution. The LFP electrode was pulled from a $1 \mathrm{~mm}$ outer diameter quartz capillary on a laser-based pipette puller (P2000, Sutter Instruments) to a fine tip, which was then manually broken back to a diameter of $\sim 10 \mu \mathrm{m}$. The LFP electrode was filled with sterile saline.

We deployed a modified Autopatcher robot (Kodandaramaiah et al., 2012) to perform whole-cell patch-clamp recordings in current-clamp mode. Series resistance and membrane potential were continuously monitored. Membrane voltage was acquired at $20 \mathrm{kHz}$. Simultaneously, LFP was acquired at $20 \mathrm{kHz}$ and was bandpass filtered ( $1 \mathrm{~Hz}$ to $1 \mathrm{kHz}$ ). Some cells were injected with small amounts of holding current (up to $-100 \mathrm{pA})$.

Repeat-finding algorithm. The inclusion criteria for the current-clamp membrane voltage recordings were long duration ( $\geq 300 \mathrm{~s}$ ), holding current $<100 \mathrm{pA}$, steady membrane potential $(<5 \mathrm{mV}$ drift over the duration of the recording), resting membrane potential of less than $-55 \mathrm{mV}$, spike amplitude $>50 \mathrm{mV}$, and access resistance $<100 \mathrm{M} \Omega$. In the recordings that passed these criteria, spikes were detected and fully extracted (1.5 ms before the spike and $4.5 \mathrm{~ms}$ after) and replaced with $\mathrm{NaN}$ (nota-number) values. Recordings with any burst firing (defined as three or more spikes occurring within $15 \mathrm{~ms}$ ) were then excluded because bursts of spiking resulted in extended stretches of $\mathrm{NaN}$ values, which would bias the repeat-finding algorithm and the relationship between repeats and spike rate. Approximately one-half of the otherwise viable recordings were excluded due to this criterion.

To find repeats, recordings were downsampled to $2 \mathrm{kHz}$ to increase processing speed. They were then split into template segments of duration 300,900 , or $2700 \mathrm{~ms}$, with an overlap of 100,300 , and $300 \mathrm{~ms}$, respectively. Each template was then correlated to the remainder of the recording. This produced a correlation trace of the same duration as the original recording. Peaks above a correlation value [Pearson correlation coefficient $(r)]$ of 0.8 were identified and their temporal locations stored. We chose a threshold of $r=0.8$ because we found that the repeats looked qualitatively similar to those found in previous studies (Ikegaya et al., 2004; Mokeichev et al., 2007). In addition, testing of statistical significance of the repeats was performed with higher correlation coefficients $(r=0.84,0.86,0.90)$ with no difference in the resulting conclusions (data not shown). A peak that occurred within $0.5 \mathrm{~s}$ of a previous peak was discarded to reduce overlap. Repeats were constructed from these peak locations such that the center of the peak was the center of the repeat. A single template in many cases had multiple repeats. This repeat-finding algorithm was parallelized and performed on a high-performance computing cluster for all original and surrogate recordings.

Repeat characterization. We characterized the repeats using SDs and oscillatory activity. The SD of all repeats was grouped and compared with 100 randomly chosen nonrepeat segments of the recording. Oscillation activity was quantified by calculating the root mean square deviation of the spectral density from the $1 / \mathrm{fline}$, a metric we termed $D$. A large value for $D$ indicates large oscillatory activity. We confirmed this metric with the oscillatory index (OSI) metric (Mokeichev et al., 2007); briefly, it is a unitless quantity representing the amplitude of the first noncentered peak of an autocorrelation plot. Therefore, a high OSI represents a high degree of oscillatory activity, regardless of its frequency.

To detect theta oscillations in the LFP, the LFP signal was bandpass filtered for theta $(6-12 \mathrm{~Hz})$ and delta $(1-4 \mathrm{~Hz})$, and a theta/delta amplitude envelope ratio was computed. Recording segments where the ratio exceeded 1 SD above mean for at least $1 \mathrm{~s}$ were considered theta periods. To detect SWRs, the LFP was first bandpass filtered $(150-250 \mathrm{~Hz})$, and an amplitude envelope was calculated. Recording segments where the amplitude envelope exceeded 4 SDs above mean for at least $15 \mathrm{~ms}$ were considered SWRs. Since spiking events may happen before and after the detected SWR event (Davidson et al., 2009; Dragoi and Tonegawa, 2011), portions of the recording $400 \mathrm{~ms}$ before and after the envelope peak were considered part of the SWR. Theta and SWR periods were also visually inspected to validate these detection algorithms. To determine whether repeats occurred preferentially during LFP events (theta oscillations and SWRs), we calculated the total fraction of repeats occurring during a network state and compared it to the fraction of repeats occurring outside that network state. We also tested repeat reoccurrence: given that templates occur during particular network states, we calculated the probability of their repeats occurring during the same or a different network state. For example, the fraction of repeats that occurred during theta that had templates that occurred during SWRs (SWR $\rightarrow$ theta) was calculated by (1) identifying the templates that occurred during SWRs, (2) compiling all the repeats of those templates, and (3) calculating the fraction of those repeats that occurred during theta. A template or repeat was considered to occur during a network state whether $>50 \%$ of that template or repeat occurred during the network state. Repeats could also occur during certain network states by chance; this chance level was calculated to be time spent in the network state divided by the total recording duration.

To compare the spectral content of templates, repeats, and nonrepeats, we computed the spectral angle (Yuhas, 1992). The spectral angle converts spectral densities of two signals into an $f$-dimensional vector, where $f$ is the number of sampled frequencies, and computes the solid angle between the two vectors. Spectral angles can take values between 0 and $\pi / 2$, where values closer to 0 indicate a higher degree of spectral similarity. Power in specific frequency bands was calculated by bandpass filtering the recording to the relevant frequencies using finite impulse response filters and calculating the mean of the squared amplitude of the filtered signal over the repeat duration $(900 \mathrm{~ms})$. To isolate low-frequency components, we used a low-pass digital Butterworth filter with a cutoff frequency of $100 \mathrm{~Hz}$. To isolate high-frequency components on the order of $\leq 10 \mathrm{~ms}$, we used a high-pass digital Butterworth filter with a cutoff frequency of $100 \mathrm{~Hz}$. To compare the incidence of high-frequency fluctuations between repeats and nonrepeats, segments of the recording that were not parts of any repeat were chosen randomly. The number of nonrepeat segments chosen was equal to the number of repeats found for each template.

Significance testing of repeats. We used three surrogate recording generation techniques: (1) synaptic input model; (2) phase shuffling; and (3) interval shuffling (Mokeichev et al., 2007). For the synaptic model, we modified a passive stochastic synaptic input model (Paninski et al., 2012) using the following equation:

$$
\begin{aligned}
V(t+d t)=V(t)+\left(g_{l}\left(V_{l}-V(t)\right)+g_{e}(\right. & \left.V_{e}-V(t)\right) \\
& \left.+g_{i}\left(V_{i}-V(t)\right)\right) d t+\sigma,
\end{aligned}
$$

where $V$ is the membrane voltage at time $t ; g_{v}, g_{e}$, and $g_{i}$ are leak, excitatory, and inhibitory conductances; $V_{l}, V_{e}$, and $V_{i}$ are the respective reversal potentials; and $\sigma$ is Gaussian white noise, added to model the recording noise. The excitatory and inhibitory conductances are defined the same way as in the study by Paninski et al. (2012); the only difference was in the definition of instantaneous conductances $N_{e}(t)$ and $N_{i}(t)$ that contribute to $g_{e}$ and $g_{i}$. For excitatory $(e)$ and inhibitory $(i)$ conductances, we defined $N_{e}(t)\left(N_{i}(t)\right)$ as the sum of 10 spike trains with spike rate $r_{e}\left(r_{i}\right)$, convolved with an $\alpha$ conductance function with maximum conductance $g_{e \_ \text {max }}\left(g_{i \_ \text {max }}\right)$ and time constant $\tau_{e}\left(\tau_{i}\right)$. We used simulated annealing to optimize seven of these variables, which were determined empirically to be most relevant to recapitulating aspects of our model $\left(V_{l}, g_{l}, r_{e}, g_{e-\max }\right.$ $\left.g_{i \_ \text {max }}, \tau_{e}, \tau_{i}\right)$. The error function for the simulated annealing algorithm 
was a combination of the difference in membrane voltage distributions and the difference in the power spectra between the real data and model output. Thus, the algorithm produced surrogates that were markedly similar in power spectrum and membrane voltage to original recordings. For phase shuffling, the phases of the signal in the frequency domain were shifted by a random quantity, and a recording was reconstructed by performing an inverse fast Fourier transform. For interval shuffling, two voltage levels were defined (one-third and two-thirds of voltage density) and the recording was split into segments that started and ended at these crossing points. These subsegments were then restitched, ensuring that two conditions are met: first, a subsegment must start at the same voltage level (either one-third or two-thirds level) and slope (up or down) of the previous subsegment to ensure smooth transitions between subsegments; second, during restitching each subsegment must be as long as possible without exceeding a maximum length parameter. The maximum length was set to either 450, 200, or $100 \mathrm{~ms}$ (see Results). For each original recording, 10 surrogates of each type (synaptic input model, phase shuffling, and interval shuffling) were generated. Due to the computational load of the repeat-finding algorithm, generating many more surrogates would not be feasible. The number of surrogates we generate here is consistent with previous studies (Mokeichev et al., 2007) and produced large effect sizes (see Fig. $5 d$ ). The surrogate/real ratio was used to determine whether more repeats occurred in the real data (ratio, $<1$ ) or in the surrogate data (ratio, $>1$ ).

Spike rate and repeat rate. Spike rate and repeat rate over time were each computed by convolution with a Gaussian kernel (SD, 5 s). To determine whether spikes occurred preferentially within repeats when accounting for periods of depolarization and periods of high variability, membrane potential fluctuations above a voltage threshold or variance threshold were isolated. For membrane voltage, we used the top $10 \%$ of each recording and $10 \mathrm{mV}$ below the lowest spike threshold for thresholds. For variance thresholds, we first used the top $10 \%$ of a running variance metric (Welford, 1962), and, second, we used the average variance of repeats. The isolated above-threshold fluctuations were then grouped according to whether they appeared as part of a repeat or not. The normalized spike rate was calculated as follows: $\left(R_{\text {repeat }}-R_{\text {nonrepeat }}\right) /\left(R_{\text {repeat }}+R_{\text {nonrepeat }}\right)$ where $R_{\text {repeat }}$ and $R_{\text {nonrepeat }}$ are the spike rates (in hertz) during the above-threshold repeats and above-threshold nonrepeats, respectively. Using the normalized spike rate metric, values $>0$ indicated spikes occurring preferentially during repeats.

Spike precision within repeats was evaluated using two methods. In the first method, precision was bootstrapped with spike shuffling. In every template-repeat pair, we found and recorded the smallest difference in spike times by calculating every pairwise difference between template spike times and repeat spike times, and taking the minimum value. If the minimum value was below a threshold of $20 \mathrm{~ms}$, we marked the spike in the repeat as a "precise" spike. As a control, we generated a random spike train with the same number of spikes as in the original recording and ran the same analysis using the original repeat data. We generated 10 shuffled spike trains for each original template-repeat pair. In the second method, we used spike jittering (Amarasingham et al., 2012). With this technique, to generate surrogate data, the time of each spike is resampled within a particular window (jitter time), which we set to $5-100 \mathrm{~ms}$ in $5 \mathrm{~ms}$ intervals. For each resampled recording, the number of precise spikes was calculated. A decrease in the number of precise spikes as a function of jitter time indicates a disruption in spike timing. A total of 100 shuffles was performed for every jitter time.

Experimental design and statistical analysis. All statistical analyses were performed using MATLAB (RRID:SCR_001622). A total of nine intracellular recordings were obtained from eight cells in seven male mice. Two of the recordings were from the same cell but were not acquired contiguously. For repeat searching and classification, all nine recordings were used. In comparing repeat occurrence in network states, only recordings with at least one epoch of the network state or at least one occurrence of repeats in the network state were considered. For repeat significance testing, all recordings that had repeats (eight of nine) were used. For analyses involving comparisons of spike rates and repeat rates, five recordings that had any spikes and any repeats were used. For spike precision analysis, four recordings that had at least one spike-containing template and repeat were used. The normality of data was assessed using the Anderson-Darling test. In normally distributed data, results are presented as the mean $\pm \mathrm{SD}$, unless otherwise specified. Otherwise, the results are presented as the median (range). Statistical details are presented in text and in figure legends. The Kruskal-Wallis test was used to test for differences in original and surrogate recording populations. Recordings were then analyzed independently using the Wilcoxon signedrank test with a Bonferroni correction to adjust for multiple surrogate types. Effect size was calculated using Hedge's $g$. A value of $p<0.05$ was considered statistically significant unless otherwise specified.

\section{Results}

Whole-cell recordings were performed to analyze the incidence of repeated membrane potential fluctuations in awake, behaving mice. Recordings were $7 \pm 1.5 \mathrm{~min}$ in duration (minimum, 4.7 min; maximum, $8.6 \mathrm{~min}$ ). Seven of the cells were putative hippocampal CA1 pyramidal cells, as distinguished by their low firing rate $(0.050 \pm 0.049 \mathrm{~Hz})$, and one cell had a higher firing rate (recording 4, 1.14 Hz). Since the results from the cell that had a higher firing rate did not appear as outliers in any of our analyses, results were pooled across all cells. The LFP was recorded simultaneously with membrane voltage to identify network states (theta oscillations and sharp-wave ripples; Fig. 1a,b). To determine whether cells showed any evidence of up and down states, the traces and their membrane voltage distributions were inspected. No cells had a clear bimodal distribution, indicating the lack of persistent up and down states (Fig. 1c).

\section{Repeated membrane potential patterns in the awake brain}

We first asked, over what timescales is repeated intracellular activity observed? To address this, we searched for repeated membrane voltage patterns on the following three timescales: 300, 900, and $2700 \mathrm{~ms}$. We found many pairs of highly similar recording segments, which we term template-repeat pairs (Fig. $1 d$ ). Repeats were found on all timescales but not in all recordings: 300-mslong repeats were found in nine of nine recordings; $900-\mathrm{ms}-$ long repeats were found in eight of nine recordings; 2700-ms-long repeats were found in two of nine recordings (Fig. 1e). Our analysis focused on 900-ms-long repeats (Fig. $1 f$ ) due to their relative abundance and their long duration relative to previously characterized repeated spiking patterns, and to better allow comparison to previous characterization of subthreshold patterns of activity in vitro and in anesthetized animals (Ikegaya et al., 2004, 2008; Mokeichev et al., 2007).Therefore, in all subsequent analyses, only the 900-ms-long segments were considered. The templaterepeat pairs exhibited a high degree of correlation (Pearson's $r$ values between 0.80 and $0.89 \pm 0.037 ; n=8$ recordings with nonzero repeats; Fig. $1 g$ ). We also found many repeats that correlated with templates from other recordings ( $n=8$ of 8 recordings with nonzero repeats; Fig. $1 h$, Table 1 ), suggesting the presence of stereotyped intracellular activity across cells in the hippocampus. Unlike in anesthetized cortex (Mokeichev et al., 2007), the number of repeats we found varied dramatically between recordings. While the median number of repeats was 135, we found anywhere from 0 (recording 6) to 7159 repeats (recording 1) in a recording. This variation was not accounted for by the differences in recording duration; with a longer recording, the number of candidate repeats is expected to grow quadratically (Mokeichev et al., 2007), which was not observed in our data (Fig. 2a)

After identifying template-repeat pairs, we asked whether the repeats shared common features that separated them from nonrepeats. First, we examined the variability in the membrane voltage, quantified as the SD of the membrane voltage because at any given baseline membrane voltage, its variability directly affects 
a

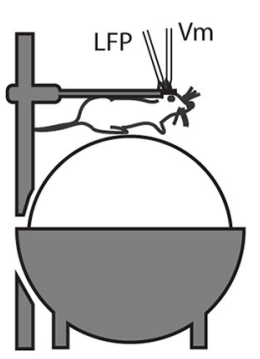

b Theta oscillations

Raw trace

Trace bandpass-filtered 6-12 Hz

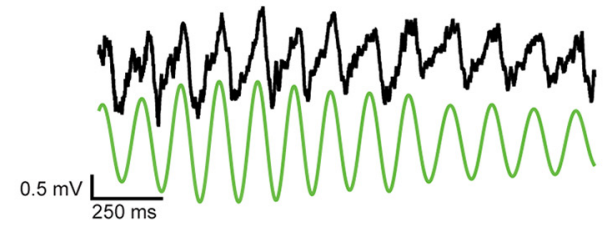

Raw trace

Sharp-wave ripples

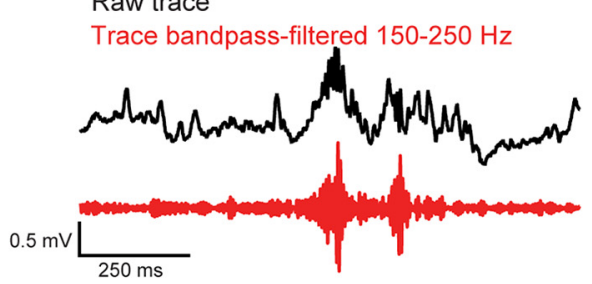

C

d

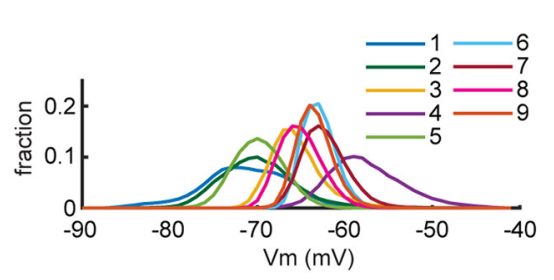

e

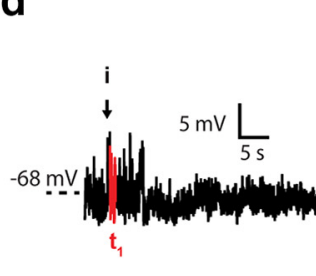

(4)

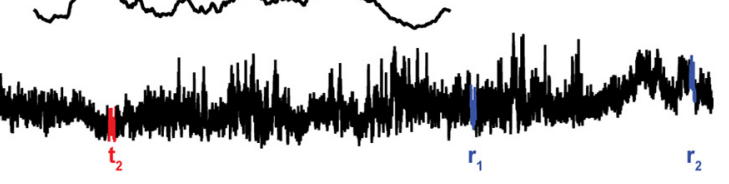

$\mathbf{f}$

template-repeat pairs from $d$

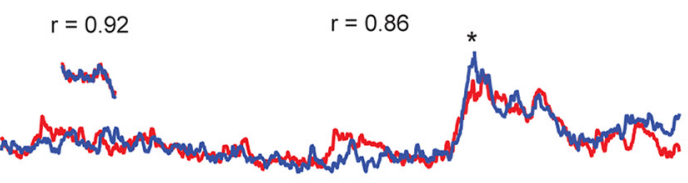

h

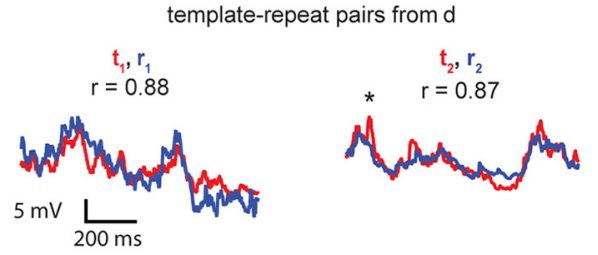

g

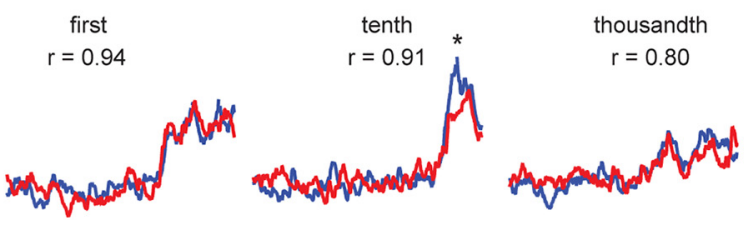

$\begin{array}{ccc}\text { template of } 4 \text { found in } 7 & \text { template of } 2 \text { found in } 1 & \text { template of } 3 \text { found in } 8 \\ r=0.89 & r=0.91 & r=0.88\end{array}$

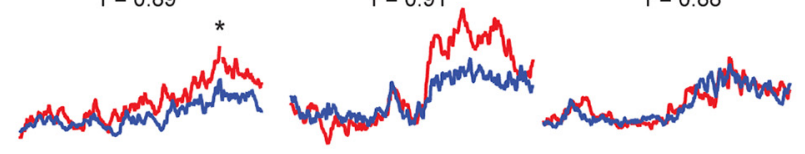

Figure 1. Repeated patterns of membrane voltage are found in CA1 of awake, behaving mice. $\boldsymbol{a}$, Experimental setup showing the awake, headfixed mouse on a floating Styrofoam ball. Membrane voltage $\left(V_{\mathrm{m}}\right)$ is recorded with a patch-clamp electrode, LFP is recorded with an extracellular electrode. $\boldsymbol{b}$, Sample LFP segments showing theta oscillations (left) and two sharp-wave ripples (right). $\boldsymbol{c}$, Voltage histograms of all recordings. $\boldsymbol{d}$, Representative recording (recording 3 ) with two template-repeat pairs (t1, template 1 ; $r 1$, repeat of template 1 ; $t 2$, template 2 ; $r 2$, repeat of template 2). Inset (i): sample trace with extracted spikes (marked with *) $\boldsymbol{e}$, Sample template-repeat pairs of 300 and $2700 \mathrm{~ms}$ duration, $r$ shown above traces indicates Pearson's $r$ between template and repeat. $\boldsymbol{f}$, Superimposed $900 \mathrm{~ms}$ template-repeat pairs from the recording in $\boldsymbol{d}$ (templates in red and repeats in blue). $\boldsymbol{g}$, Sample $900 \mathrm{~ms}$ template-repeat pairs from recording 1 rank ordered by $r$ value (i.e., the "first" pair is the most similar repeat in the recording; the "tenth" pair is the tenth best). $\boldsymbol{h}$, Sample pairs with templates from one recording with repeats found in another recording.

Table 1. Number of repeats from templates in different recordings

\begin{tabular}{|c|c|c|c|c|c|c|c|c|c|}
\hline \multirow[b]{2}{*}{ Template from } & \multicolumn{9}{|c|}{ Repeats in } \\
\hline & 1 & 2 & 3 & 4 & 5 & 6 & 7 & 8 & 9 \\
\hline 1 & 7159 & 928 & 1218 & 1500 & 221 & 0 & 269 & 206 & 26 \\
\hline 2 & 954 & 1012 & 505 & 790 & 218 & 0 & 391 & 263 & 49 \\
\hline 3 & 1208 & 483 & 412 & 693 & 49 & 0 & 127 & 76 & 10 \\
\hline 4 & 0 & 726 & 627 & 1062 & 119 & 0 & 230 & 172 & 28 \\
\hline 5 & 224 & 208 & 72 & 139 & 53 & 0 & 64 & 61 & 5 \\
\hline 6 & 34 & 4 & 0 & 4 & 0 & 0 & 0 & 0 & 0 \\
\hline 7 & 242 & 284 & 127 & 217 & 46 & 0 & 121 & 96 & 8 \\
\hline 8 & 268 & 266 & 113 & 208 & 41 & 0 & 68 & 111 & 6 \\
\hline 9 & 58 & 80 & 19 & 40 & 0 & 0 & 20 & 14 & 8 \\
\hline
\end{tabular}

Templates from (row) recording are used to find repeats in (column) recording. The numbers in the diagonal (e.g. $\{1,1\},\{2,2\})$ are the number of repeats in each recording (Fig. 2a).

the probability of spiking, with a larger SD corresponding to an increased likelihood that the membrane voltage will rise enough to trigger a spike (Bennett et al., 2013). In the extreme case, with an $\mathrm{SD}$ of 0 , the neuron would never spike for any baseline voltage below spike threshold. At the other extreme, if the SD was very high, the neuron would very often find itself at a voltage sufficient for spike initiation. We found that repeats tended to have larger fluctuations in the membrane potential: they had higher-amplitude fluctuations than randomly chosen nonrepeat segments of the recording (Fig. $2 b, c$ ); however, they were not preferentially depolarizing or hyperpolarizing relative to the mean voltage of each recording (mean, $0.14 \pm 0.93 \mathrm{mV} ; t_{(7)}=0.44, p=0.67$, Student's $t$ test; $n=8$ recordings with nonzero repeats; effect size, 0.16 ).

Because our analysis could mistakenly identify oscillations in the membrane potential as repeats, we asked whether the templaterepeat correlation was the result of oscillations. This was tested by correlating the Pearson's $r$ value of the template-repeat pairs to the deviation of the recording spectrum from $1 / f(D$ metric). No correlation was found (Fig. $2 d, e$ ); this was confirmed with the OSI metric (median Pearson's $r$ of OSI vs repeat correlation: $-0.04 ; p=0.64$, Wilcoxon signed-rank test; $n=8$ recordings; $\mathrm{df}=7$; effect size, 0.14 ). Thus, we conclude that repeated patterns were not due to oscillations in the membrane potential.

To determine whether repeats were more likely to occur during a particular network state or behavioral state (e.g., immobility vs locomotion), we examined the occurrence of repeats during theta oscillations (correlated with movement) and SWRs (which occurred when the animal was still) in the LFP. We reasoned that (1) repeats would occur preferentially during some network states over others and (2) templates would have repeats that re- 


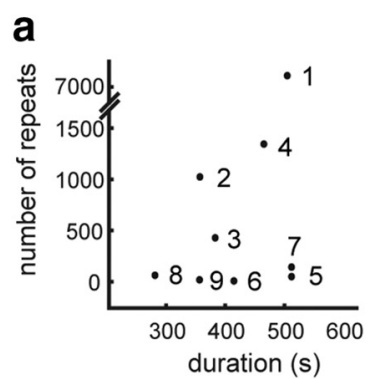

b

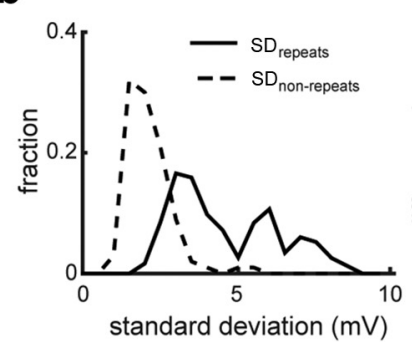

e

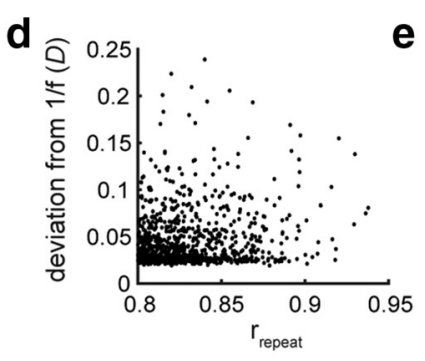

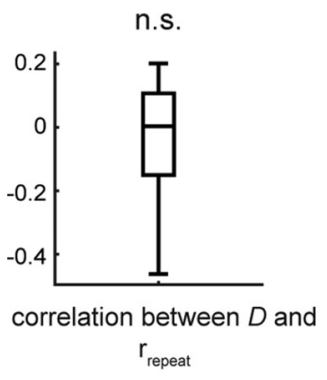

C

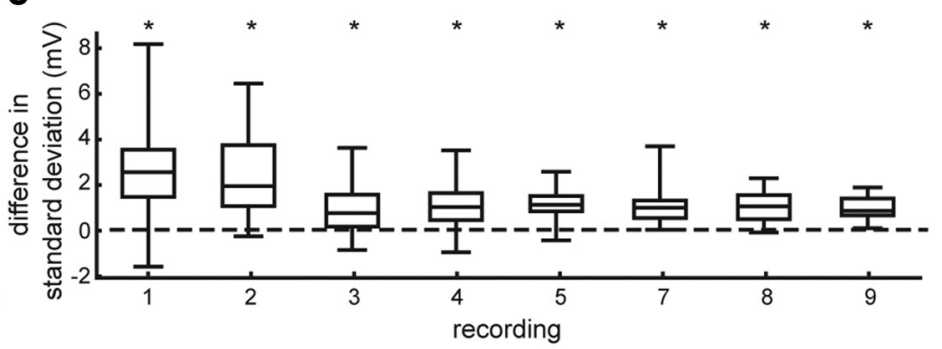

f

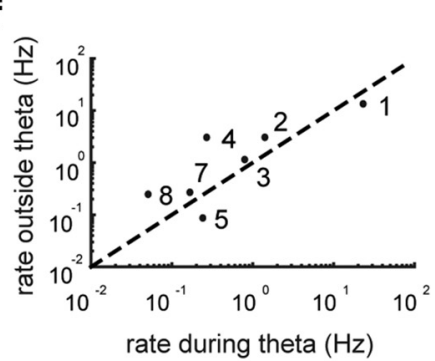

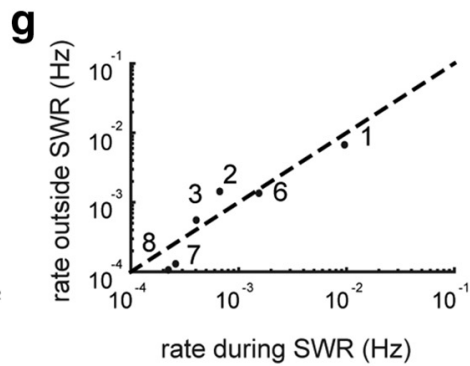

Figure 2. Repeat classification. $\boldsymbol{a}$, Relationship between recording duration and the number of repeats (no correlation; Pearson's $r=0.41 ; p=0.27, n=9$ recordings). $\boldsymbol{b}$, Sample histogram of standard deviations (SD) for repeats (solid) and 100 randomly chosen nonrepeats (dashed) in one recording (recording 1). In this example, the difference between the SD of the repeats and the nonrepeats has a median of $2.55 \mathrm{mV}$ (range, -1.59 to $8.17 \mathrm{mV}$ ). c, Difference in SDs of repeats and nonrepeats for each recording. In all recordings, the median difference was $>0 \mathrm{mV}$ (minimum, $0.76 \mathrm{mV}$; maximum, $2.56 \mathrm{mV} ;{ }^{*} p<0.01$, Wilcoxon signed-rank test; $n=8$ recordings with nonzero repeats; df $=7$; effect sizes: $1.57,1.47,0.99,1.29,2.2,1.38,1.65$, and 1.74$)$, indicating that repeats tend to have a higher SD than nonrepeats. $\boldsymbol{d}$, Sample scatterplot of the repeat correlation (Pearson's $r$ ) value and spectrum deviation from 1/f(D) from recording 2.e, Correlation (Pearson's $r$ ) between $D$ and $r_{\text {repeat }}$ for every cell. On the population level, the mean correlation is not different from $0(r=-0.04 \pm 0.22 ; p=0.60$ (n.s.); $n=8$ recordings with nonzero repeats; $\mathrm{df}=7$ ). $\boldsymbol{f}$, $\boldsymbol{g}$, Occurrence of repeats within and outside of theta $(\boldsymbol{f})$ and SWR $(\boldsymbol{g})$ activity in the LFP. Recordings with at least one identified theta oscillation or SWR episode are shown. Repeats did not occur preferentially during theta oscillations ( $p=0.47$, Wilcoxon signed-rank test; $n=7, \mathrm{df}=7$, effect size $=0.27$ ) or SWRs ( $p=0.69$, Wilcoxon signed-rank test; $n=6$, df $=6$; effect size $=0.007$ ). Box plots show median and first/third quartiles. Whiskers show the data range. $r_{\text {repeat }}$ repeat correlation (Pearson's $r$ ).

occur preferentially during the same network state as the templates. Surprisingly, neither prediction was supported by the results. First, the overall rate of repeat occurrence was not significantly different during theta and SWR occurrence (Fig. 2f,g). Second, templates occurring during a particular network state in the LFP had repeats reoccur during that same network state at chance level (theta $\rightarrow$ theta: $p=0.94, n=6, \mathrm{df}=6$, effect size $=$ 0.022 ; SWR $\rightarrow$ SWR: $p=0.84, n=8, \mathrm{df}=8$, effect size $=0.26$; Wilcoxon signed-rank test). Similarly, templates that occurred during one state had chance levels of repeats in the other state (theta $\rightarrow$ SWR: $p=0.56 ; n=6 ; \mathrm{df}=6$, effect size $=0.15 ; \mathrm{SWR} \rightarrow$ theta: $p=0.95, n=8, \mathrm{df}=8$, effect size $=0.19$; Wilcoxon signed-rank test). Thus, we found that repeat occurrence was independent of network state.

We then asked whether there are specific frequencies that are uniquely shared among intracellular repeats. We first computed the spectral densities of all templates, their repeats, and an equal number of nonrepeats. The spectral content of templates and their repeats was similar, as expected (Fig. $3 a$, left). In contrast, template spectral content differed from nonrepeat spectral content (Fig. 3a, right). To quantify this difference, we computed the spectral angle between the spectral density of the template and that of repeats and nonrepeats. The analysis confirmed that the templates were more similar (lower spectral angle) in their spectral content to their repeats than to nonrepeats (Fig. 3b). We then asked what particular frequency bands are different between repeats and nonrepeats. Repeats tended to have slow fluctuations; therefore, we examined power in the $3-6 \mathrm{~Hz}$ range, as well as theta $(6-10 \mathrm{~Hz})$ and gamma $(30-50 \mathrm{~Hz})$, prominent oscillations in the hippocampal circuit. The low-frequency $(3-6 \mathrm{~Hz})$ content in repeats was significantly higher than that in nonrepeats, while theta $(\sim 6-10 \mathrm{~Hz})$ and gamma $(\sim 30-50 \mathrm{~Hz})$ oscillations were not significantly different (Fig. $3 c$ ).

Although we found that slow fluctuations were more prominent in repeats, we wondered whether there were also higher-frequency $(>100 \mathrm{~Hz})$ components that repeat with high precision. To address this, we filtered the recordings to isolate low-frequency $(<100 \mathrm{~Hz})$ or high-frequency $(\geq 100 \mathrm{~Hz})$ components from the membrane voltage. As expected, in the traces filtered to isolate low-frequency membrane potential fluctuations, template-repeat correlations were high (Fig. 4a,b, middle traces). However, even in the traces filtered to isolate high-frequency fluctuations at the $10 \mathrm{~ms}$ timescale, the correlation between templates and repeats was present (Fig. $4 a, b$, bottom traces). We quantified the correlation between templates and repeats and compared it to the correlation between templates and 100 randomly chosen nonrepeats, and found that even on the millisecond timescale $(f \geq 100 \mathrm{~Hz})$, the correlation of template-repeat pairs was significantly higher than that between templates and nonrepeats (Fig. 4c). In short, the repeated activity we discovered had repeated features on the $10 \mathrm{~ms}$ timescale.

\section{Establishing significance of repeated patterns using statistical controls}

We then aimed to determine whether these repeated patterns occur more often than would be expected by chance given that similarities in signal segments are known to emerge randomly with sufficiently long analog time series data. To address this challenge, Mokeichev et al. (2007) previously developed techniques to generate "surrogate" recordings, or time series data that mimic the overall dynamics of the actual recorded intracellular activity, but are generated stochastically. If the number of repeats in the surrogate recordings is smaller than the number of repeats 


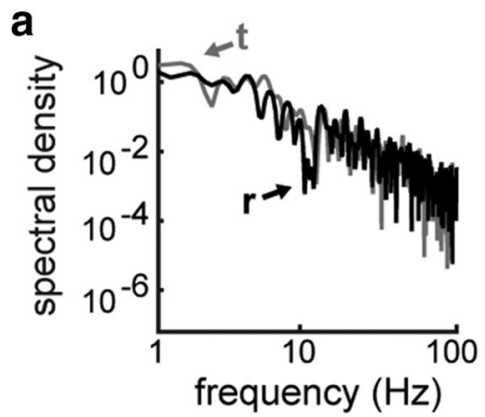

C

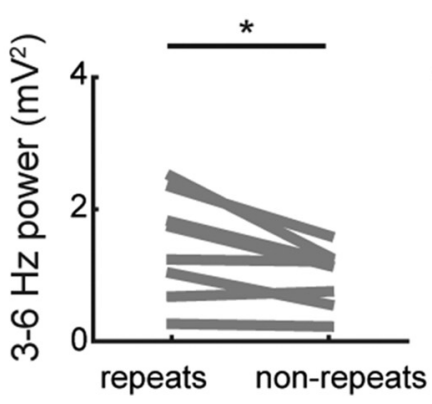

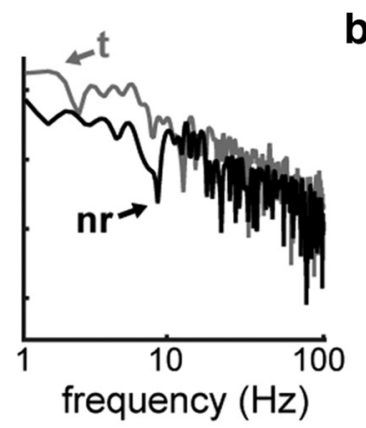
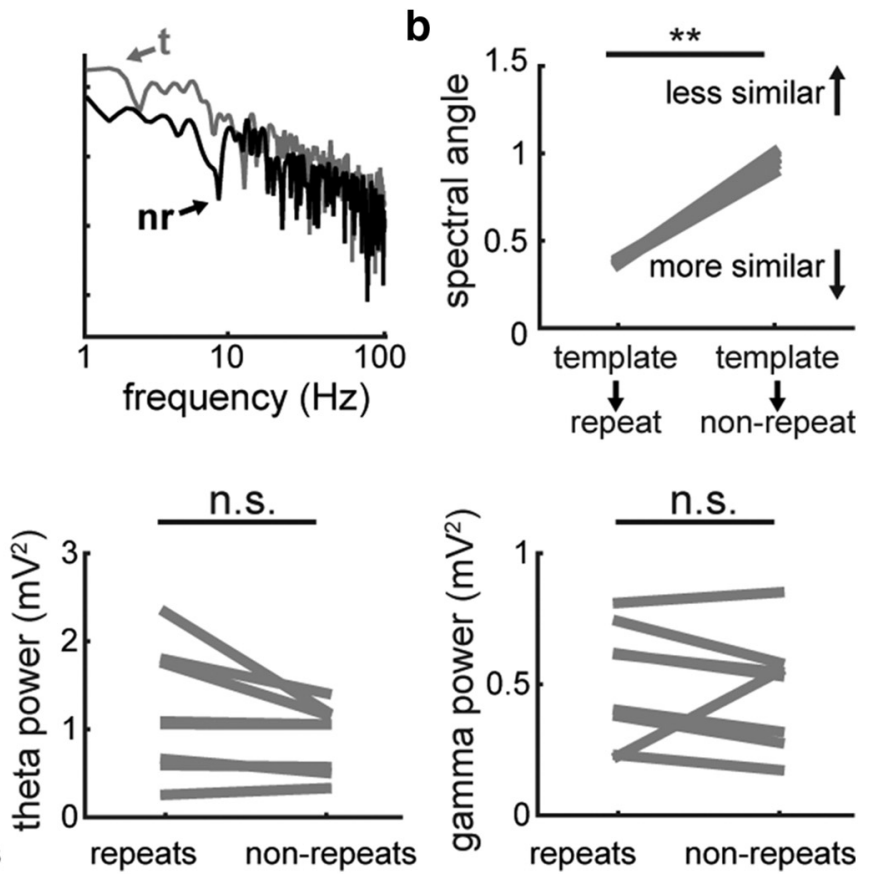

Figure 3. Spectral characteristics. $\boldsymbol{a}$, Left, Representative spectral density of a template (t; gray) and one of its repeats $(r$; black). Right, Spectral density of template and randomly chosen nonrepeat (nr; black). $\boldsymbol{b}$, Spectral angle of template-repeat pairs is significantly lower than that of template-nonrepeat pairs $\left(n=8\right.$ recordings with nonzero repeats; $\mathrm{df}=7$, ${ }^{* *} p<0.001$, effect size $=3.2$; Student's paired $t$ test). $c$, Power in specific frequency bands of repeats and nonrepeats. Repeats had significantly higher power in the low-frequency range ( $3-6 \mathrm{~Hz}$ ) but not in theta or gamma bands ( $n=8$ recordings with nonzero repeats; $\mathrm{df}=7,{ }^{*} p=0.022$, effect size $=0.57, p>0.09$ (n.s.), effect size $=0.36$ for theta, effect size $=0.10$ for gamma; Student's paired $t$ test).

in the original recordings, there is likely some deterministic structure in the real recordings that cannot be recapitulated by stochastic processes. We used the following three techniques to generate 10 surrogate recordings for every recording: (1) a stochastic model; (2) phase shuffling; and (3) interval shuffling (for details, see Mokeichev et al., 2007; Fig. 5a,b). For the stochastic model, we simulated a membrane potential recording such that it matched the duration, membrane voltage distribution, and power spectrum of each original recording. For this surrogate type, seven of eight recordings had significantly fewer repeats in the surrogate data than in the original data (Fig. $5 c, d$, blue). For phase shuffling, we generated surrogate data by transforming the original recording into the frequency domain, randomizing the phase components, and converting back to the time domain. For this surrogate type, six of eight recordings had significantly fewer repeats in the surrogate data than in the original (Fig. $5 c, d$, green). For interval shuffling, we divided each recording in the time domain along two voltage levels and assembled it back in a random order, preserving contiguous segments up to $100 \mathrm{~ms}$ in duration. For this surrogate type, eight of eight recordings had significantly fewer repeats in the surrogates than in the original data [Fig. $5 c, d$, black, interval shuffled $(100 \mathrm{~ms})]$. However, this surrogate type was highly sensitive to the duration of preserved contiguous segments. When using the value $450 \mathrm{~ms}$ (equal to the duration of the searched repeat divided by two, as in the study by Mokeichev et al., 2007), only one of eight recordings had significantly fewer repeats in the surrogates than in the original data (Fig. 5e). In fact, in many of these interval-shuffled recordings, the repeat-finding algorithm found many more repeats in the surrogate data than in the original.

Because of the manyfold increase in the number of detected repeats in the interval-shuffled $(450 \mathrm{~ms})$ surrogates, we reasoned that the interval-shuffling procedure could be subtly adding structure to the recording, thereby creating repeats. We found that the key contributor to the increase in the number of detected repeats following interval shuffling is a slow baseline variation that is often seen in awake intracellular recordings (Steriade et al., 2001). The interval-shuffling method works by splitting the recording into short segments along two voltage levels, representing onethird and two-thirds of the voltage distribution. The short segments are kept contiguous until they exceed a duration threshold, creating long segments, which are then "restitched" along the one-third and two-thirds levels. With slow baseline variation, the one-third and two-thirds levels become sufficiently separated such that there are few transition segments (i.e., segments that start on the one-third level and end on the two-thirds level or vice versa). Instead, there are comparatively more segments that start and end on the same level. This makes it likely that the intervalshuffled recording becomes structured like a noisy digital signal, with the one-third and two-thirds levels serving as the "high" and "low" levels of the signal (Fig. $5 f$ ). To test this hypothesis, we injected one template and its repeats (Fig. $5 g$ ) into a surrogate recording (Fig. 5h) to create a new artificial recording with a known number of additional repeats (Fig. 5i,j). As expected, the interval-shuffling ( $450 \mathrm{~ms}$ ) method added many more repeats to each recording (four of five artificial recordings had significantly more repeats in the surrogates than in the artificial recording; Fig. $5 k$ ). While it is possible to remove baseline variation from the recording, this could eliminate important slow components from the signal such as gradual membrane potential changes. Instead, we changed the length of the longest possible contiguous segment from $450 \mathrm{~ms}$ (the value close to the original value used by Mokeichev et al., 2007) to $100 \mathrm{~ms}$. This had the effect of adding more transition segments and largely removing the "digital-like" structure we saw with interval shuffling, while still preserving the overall dynamics of the recorded data [Fig. $5 a$, interval (100 ms)]. With a maximum interval of $200 \mathrm{~ms}$, six of eight recordings had significantly fewer repeats in the surrogates than in the original 
a

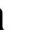

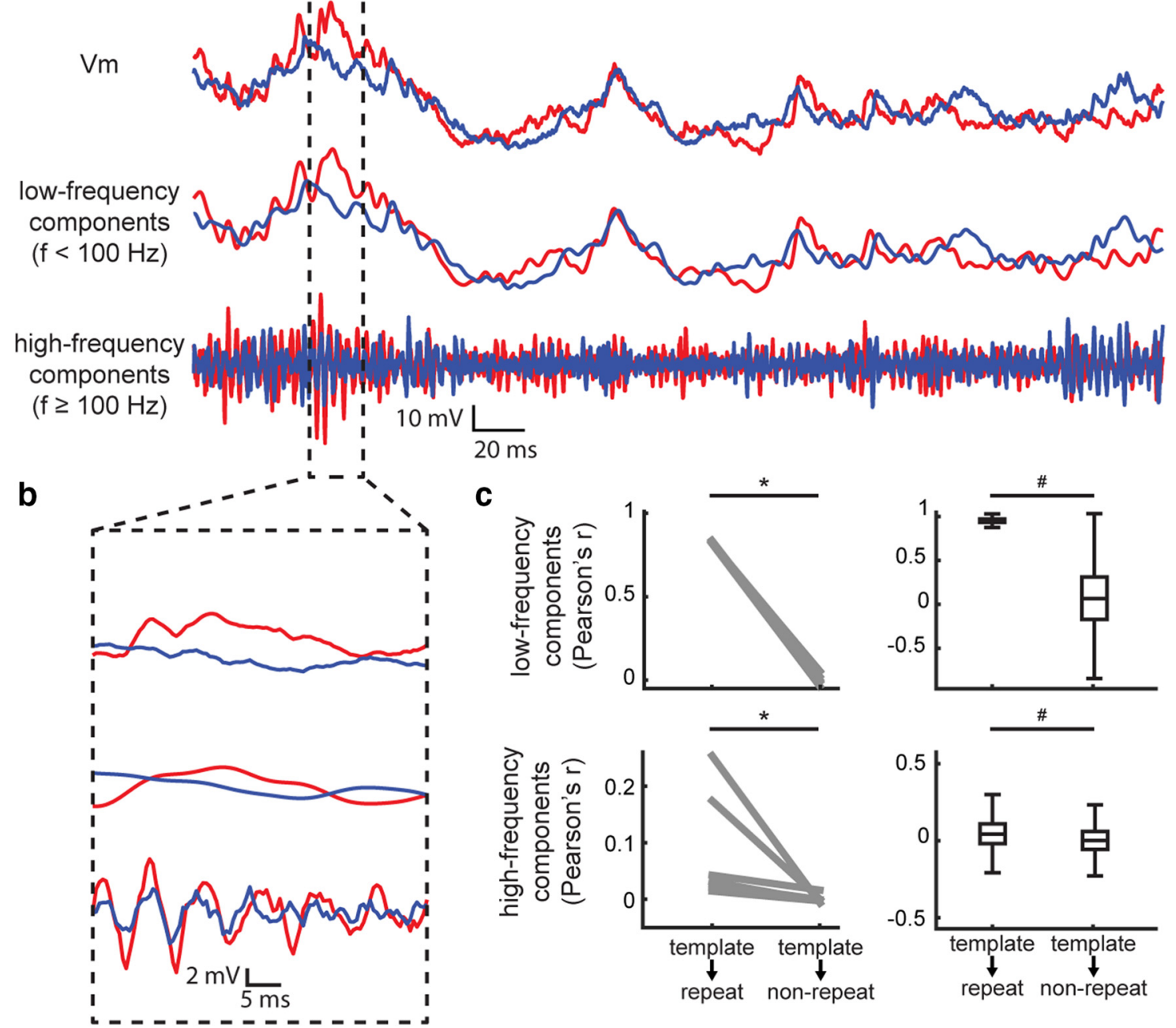

Figure 4. Precision in high frequencies of template-repeat pairs. $\boldsymbol{a}$, Sample template (red)-repeat (blue) pairs from a recording (top) filtered $<100 \mathrm{~Hz}$ to reveal slow fluctuations (middle) and $>100 \mathrm{~Hz}$ to reveal fluctuations on the $10 \mathrm{~ms}$ timescale and lower (bottom). In this example, the template-repeat pairs are correlated as follows: unfiltered membrane potential, $r=0.82$; membrane potential filtered at $<100 \mathrm{~Hz}, r=0.82$; membrane potential filtered at $>100 \mathrm{~Hz}, r=0.15$. $\boldsymbol{b}$, Inset showing the template (red) and repeat (blue) for the unfiltered trace (top), low frequencies (middle), and high frequencies (bottom). Repeat correlation is preserved at high frequencies. $c$, Low-frequency (top row) and high-frequency (10 ms timescale, bottom row) correlations are higher for template-repeat pairs than for randomly chosen template-nonrepeat pairs. Left column shows comparison of average $r$ values per recording $[n=8$ recordings with nonzero repeats, $\mathrm{df}=8,{ }^{*} p<0.01$, effect size $=5.4$ (top), 0.33 (bottom); Wilcoxon signed-rank test]; right column shows aggregated $r$ values for all repeats $[n=10,083$ repeats, $\mathrm{df}=10,083$; $\# p<0.001$; effect size $=2.01$ (top), 0.15 (bottom); Mann-Whitney $U$ test]. Box plots show median and first/third quartiles. Whiskers show the data range.

data, which is similar to the results using a maximum interval of $100 \mathrm{~ms}$. We therefore concluded that the $100 \mathrm{~ms}$ interval shuffling parameter is robust. We were unable to test maximum intervals $<100 \mathrm{~ms}$ because such short intervals led to significant decreases in the number of usable subsegments for restitching. In the interval-shuffling algorithm, recording segments that do not cross the one-third and two-thirds voltage levels over the duration of the maximum length parameter $(100 \mathrm{~ms})$ are discarded and not used for restitching, as was done in a previous study (Mokeichev et al., 2007). For example, changing the parameter to 50 ms leads to the discarding of the majority $(>60 \%)$ of the recording.

\section{Correlating repeated patterns with spiking activity}

We next examined whether repeats were related to the spiking output of the cell. This could indicate that the repeats are an important feature of activity propagation in the network. No overt relationship between the number of spikes and the number of repeats in a recording was observed (Pearson's $r=0.08 ; p=$
$0.84, n=9$, $\mathrm{df}=8$ ); but a temporal relationship within recordings could still exist. We analyzed the relationship between repeat occurrence in the recording and the spiking activity of the cell and found them to be correlated. The "repeat rate" was defined as the rate at which repeats occur in the recording regardless of their parent template. This rate was compared with the spike rate of the cell, revealing a high level of correlation (median, 0.60; range, -0.16 to 0.63 ; Fig. $6 a, b$ ). When spikes were shuffled over the entire recording, this correlation disappeared (median, 0.06; range, -0.33 to $0.10 ; n=5$ recordings with number of spikes $>0$ and number of repeats $>0$ ). Treating repeats as a rate is useful for direct comparison to the spike rate but could be misleading due to the occurrence of overlapping repeats (Fig. 6a) from different templates, which typically signify that the repeated activity lasts longer than $900 \mathrm{~ms}$. To control for this, we looked for spikes occurring during any repeat. If we restricted the search to nonoverlapping time windows, we would exclude these longer repeats, and, as a result, we may not accurately identify which spikes occur during repeats. Instead, we classify each time point in the 
a original
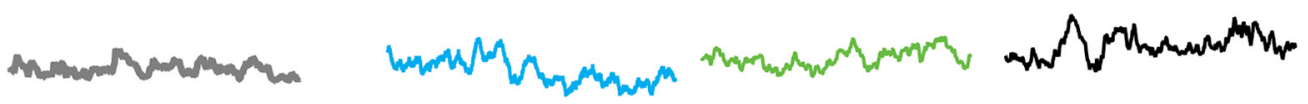

b

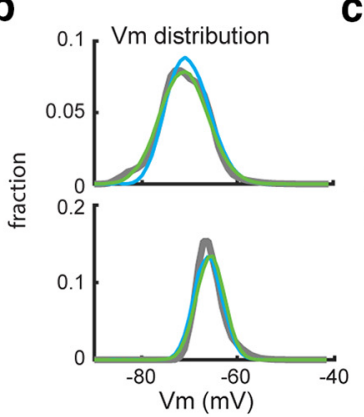

C
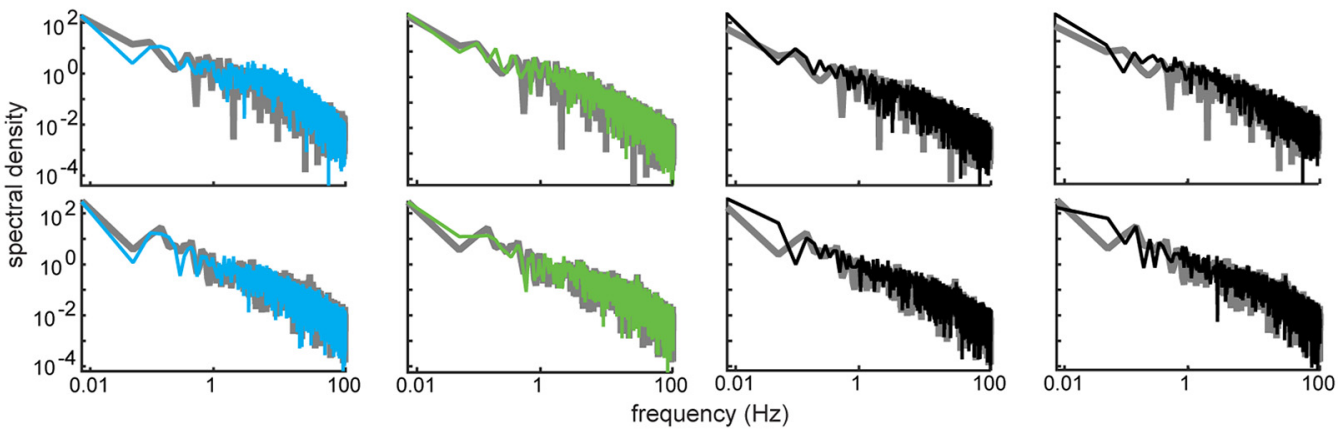

d
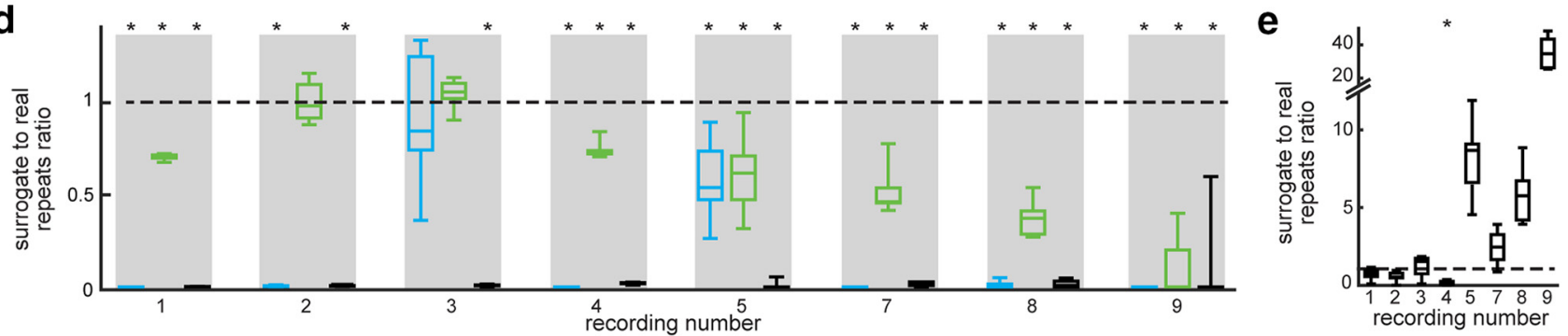

f

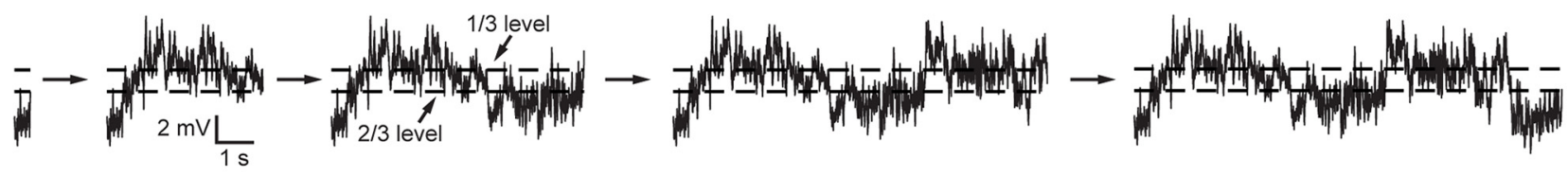

g

h

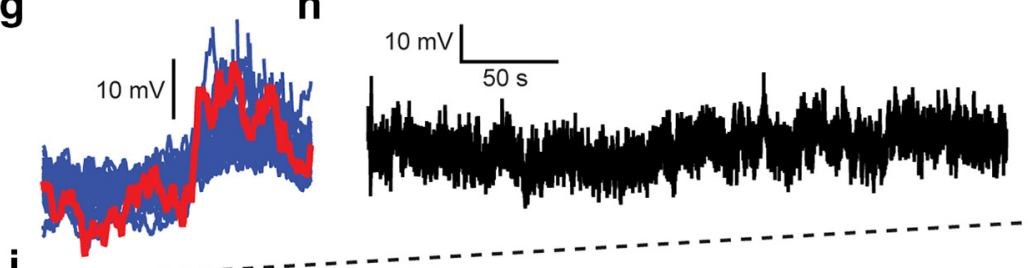

I

phase-shuffled recording + superimposed repeats
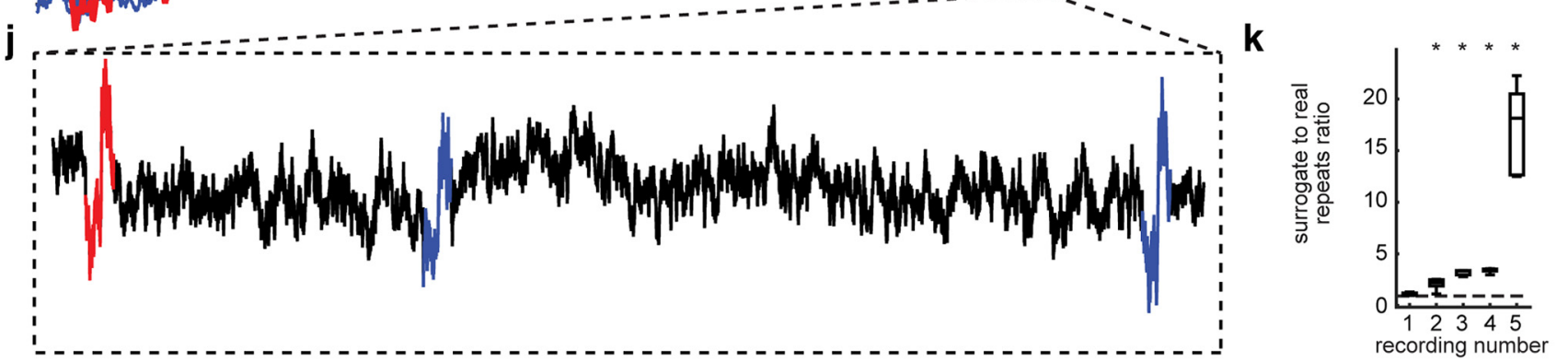

Figure 5. Statistical significance of repeated activity. $\boldsymbol{a}$, Samples ( $900 \mathrm{~ms}$ duration) of original recordings and their surrogates. Two sample recordings (recording 1 , top; recording 5 , bottom) are shown thay have different membrane potential distributions to show that the three surrogate data types can mimic the membrane voltage distribution of the original recordings. $\boldsymbol{b}$, Membrane voltage $\left(V_{m}\right)$ distributions of sample recordings (recording 1, top; recording 5, bottom). The distribution for interval-shuffled recordings is identical to that of original recordings and is not shown. c, Spectral density plots of the surrogates. Spectral density of original recording is superimposed on all plots (gray). $\boldsymbol{d}$, Ratio of the number of repeats found in the surrogate recordings to those found in original recordings ( $n=10$ surrogates of each type for each of 8 recordings with nonzero repeats). The surrogate recordings used are "model" (blue), "phase shuffled" (green), and "interval shuffled ( $100 \mathrm{~ms}$ )" (black). Recording 6 is omitted because it had no repeats. Dashed line indicates a ratio of 1 (number of real repeats = number of surrogate repeats). A ratio of $<1$ indicates more repeats found in real recordings than in their surrogates; $\mathrm{df}=9$, effect size (left to right) $=6770,123,1410,317,20,317,6.3,3.1,237$, not applicable (N/A), 46, 194, 8.2, 8.1, 57, N/A, 13, 92, 65.3, $15.7,58.1, \mathrm{~N} / \mathrm{A}, 6.6,5.6 ; p=3 \times 10^{-5}$ Kruskal-Wallis test; ${ }^{*} p<0.05 / 3$, Wilcoxon signed-rank test (with Bonferroni correction to account for three statistical tests per cell). Effect size is N/A for models that produced 0 repeats. $\boldsymbol{e}$, Ratio for interval-shuffled (450 ms) surrogates. df $=9$; effect size (left to right) $=6.02,6.52,3.83,11.8,4.52,3.76$, 4.24, 3.96; (Figure legend continues.) 
a

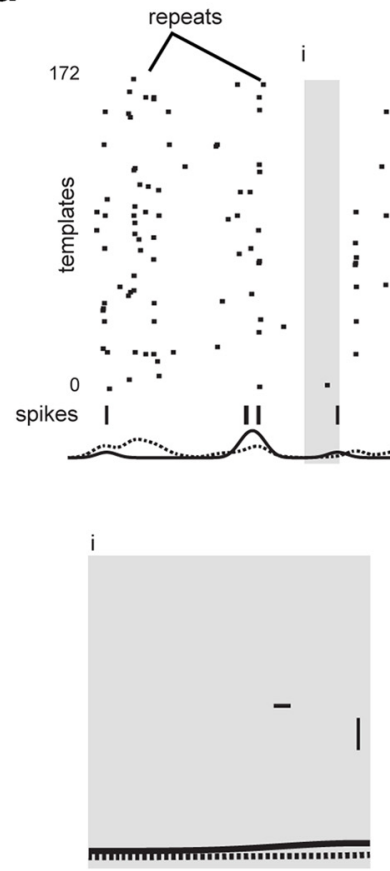

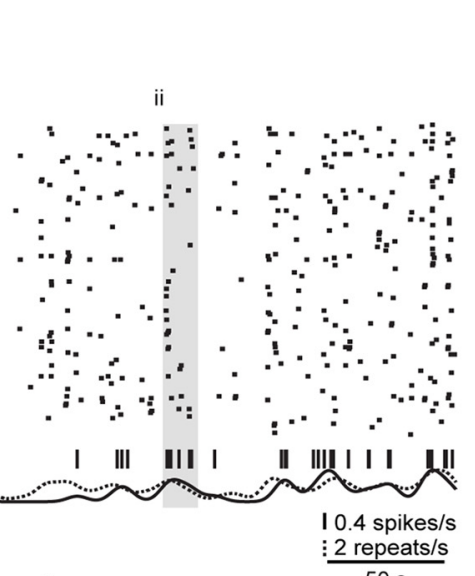

b
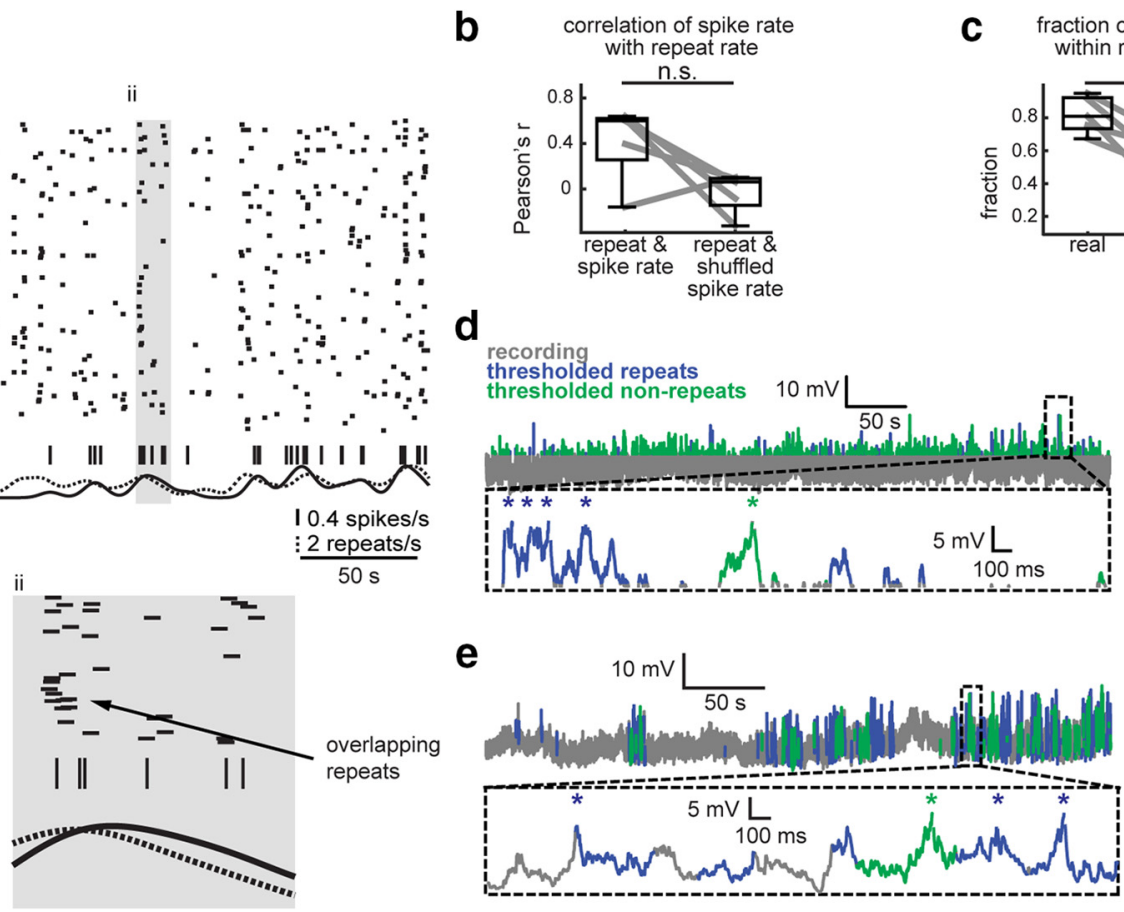

e

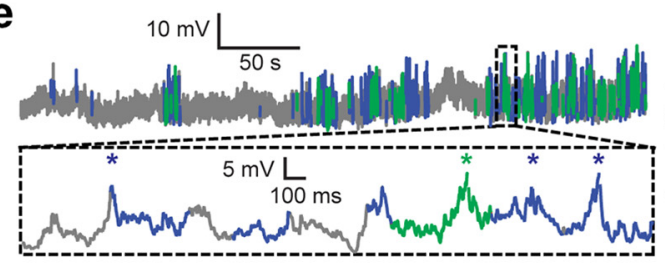

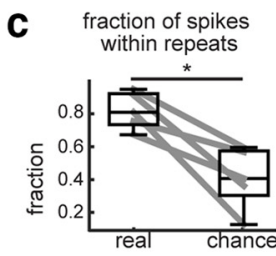
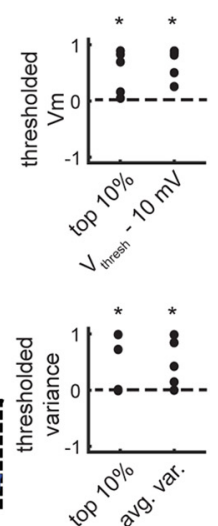

Figure 6. The majority of spikes occur during intracellular repeats. $\boldsymbol{a}$, Rastergram of repeats spikes and their occurrence rates over time for recording 3 (dotted and dashed lines, respectively). Insets highlight areas of low occurrence of spikes and repeats (1) and high occurrence (2). Arrow shows examples of overlapping repeats. $\boldsymbol{b}$, Spike rate tends to correlate with the repeat rate, an effect that disappears when spikes are shuffled. $p=0.0625$ (n.s.), Wilcoxon signed-rank test $(n=5$ recordings with the number of spikes $>0$ and the number of repeats $>0$; $\mathrm{df}=5$; effect size $=0.88$ ). c, Spikes occur within repeats at higher levels than expected by chance. Chance level is the fraction of each recording that is part of at least one repeat. ${ }^{*} p=0.0313$, Wilcoxon signed-rank test $(n=$ 5 recordings with the number of spikes $>0$ and the number of repeats $>0 ; \mathrm{df}=5$, effect size $=1.05)$. Box plots show median and first/third quartiles. Whiskers show the data range. $\boldsymbol{d}$, Left, Sample recording (recording 7) thresholded to isolate depolarizing events above the minimum spike threshold $-10 \mathrm{mV}\left(\mathrm{V}_{\text {thresh }}-10 \mathrm{mV}\right)$. Gray, Entire recording; blue, segments of repeats above the threshold; green, segments of nonrepeats above the threshold. Inset, Spikes $(*)$ occurring within (blue) and outside (green) repeats. Here, four spikes occurred within thresholded repeats and one spike occurred outside them. Right, Normalized spike rate ( -1 to 1 ) upon application of membrane voltage thresholds. Normalized spike rate $>0$ indicates more spikes occurring during thresholded repeats than during thresholded nonrepeats. Top $10 \%: t_{(4)}=3.05, p=0.019$; effect size $=1.37 ; V_{\text {thresh }}-10 \mathrm{mV}: t_{(4)}=5.36, p=0.0029$; effect size $=2.40$ (one-sample Student's $t$ test; $n=$ 5 recordings with the number of spikes $>0$ and the number of repeats $>0$ ). $\boldsymbol{e}$, Left, Sample recording (recording 3 ) thresholded to isolate high-variability events above the average variance of the repeats in the recording ("avg. var."). The color scheme is the same as in $\boldsymbol{d}$. Right, Normalized spike rate ( -1 to 1 ) upon application of the top $10 \%$ and average variance thresholds $\left({ }^{*} p<0.05\right)$. Top 10\%: $t_{(4)}=2.34, p=0.039$; effect size $=1.05$; avg. var.: $t_{(4)}=2.20, p=0.047$; effect size $=0.98$ (Student's test, $n=5$ recordings with the number of spikes $>0$ and the number of repeats $>0)$.

recording as "part of a repeat" or "not part of a repeat," so that every spike is then either part of a repeat or not. This removes issues with artificially high correlation rate due to overlapping repeats while ensuring that all spikes are taken into consideration. Indeed, a large fraction of spikes (median, 82\%; range, 68-96\%) occurred during at least one repeat (Fig. 6c).

The correlation between repeats and spiking could ostensibly be trivially explained by limitations of the repeat-finding algorithm-that is, if the algorithm was inherently predisposed to

\section{$\leftarrow$}

(Figure legend continued.) ${ }^{*} p<0.05$ Wilcoxon signed-rank test. Dashed line indicates ratio $=$ 1. Note the broken $y$-axis. $f$, Example of "digital-like" structure being added to the recording by the interval-shuffling $(450 \mathrm{~ms})$ method. Due to baseline variation in the original recording, as the surrogate signal is built, long segments congregate around the one-third and two-thirds voltage levels. $\boldsymbol{g}$, Sample extracted template (red) and its repeats (blue) from recoding 1. $\boldsymbol{h}$, Phase-shuffled surrogate of recording 1 onto which the sample extracted template is superimposed. $\boldsymbol{i}$, Superimposed recording with a known number of new repeats (number of repeats in phase-shuffled recording, 5014; number of repeats in recording with superimposed repeats, 5104). $\boldsymbol{j}$, Inset of recording with superimposed repeats. $\boldsymbol{k}$, Combined results of performing interval shuffling on phase-shuffled recordings with superimposed repeats. Dashed line indicates ratio $=1$ (number of real repeats $=$ number of surrogate repeats). The interval-shuffled ( $450 \mathrm{~ms}$ maximum duration) surrogates had threefold more repeats (median, 3.20; minimum, 1.12; maximum, $17.1 ; n=5$ recordings and $n=5$ surrogates per recording). $d f=4$; effect size (left to right) $=15.8,5.4,15.9,20.2,4.2 ;{ }^{*} p<0.05$, Wilcoxon signed-rank test. Box plots show median and first/third quartiles. Whiskers show data range. detecting spike-containing repeats, even if they were not truly highly correlated to the template. Namely, the repeat-finding algorithm could be preferentially detecting one or more of the following: (1) artifacts associated with spike extraction; (2) depolarizing events; and (3) high-variability events that produce spikes but are not necessarily highly correlated. Neither of these explanations account for the relationship we observe. To address the first limitation, we ensured that spikes are extracted completely from the recording before running the repeat-finding algorithm, making it unlikely that the algorithm is preferentially detecting some stereotyped feature of a spike (e.g., the sharp rise at the spike onset). This is evident from the fact that not every spike is a member of a template-repeat pair (in total, $n=147 / 675$ spikes are outside repeats; $22 \%$ ). To address the second limitation, we imposed a voltage threshold on the recordings and examined the membrane voltage segments above the threshold, thus isolating depolarizing events. Spikes still occurred more often within repeats than outside of them above the voltage threshold (Fig. $6 d$ ). To address the third limitation, we repeated the previous analysis with variance thresholds instead of voltage thresholds (Fig. 6e). The result we observe with the high-variability events is the same as for the depolarizing events: spikes still occurred preferentially within repeats even when only including highly variable or depolarized periods, suggesting that the relationship we observe between 
a
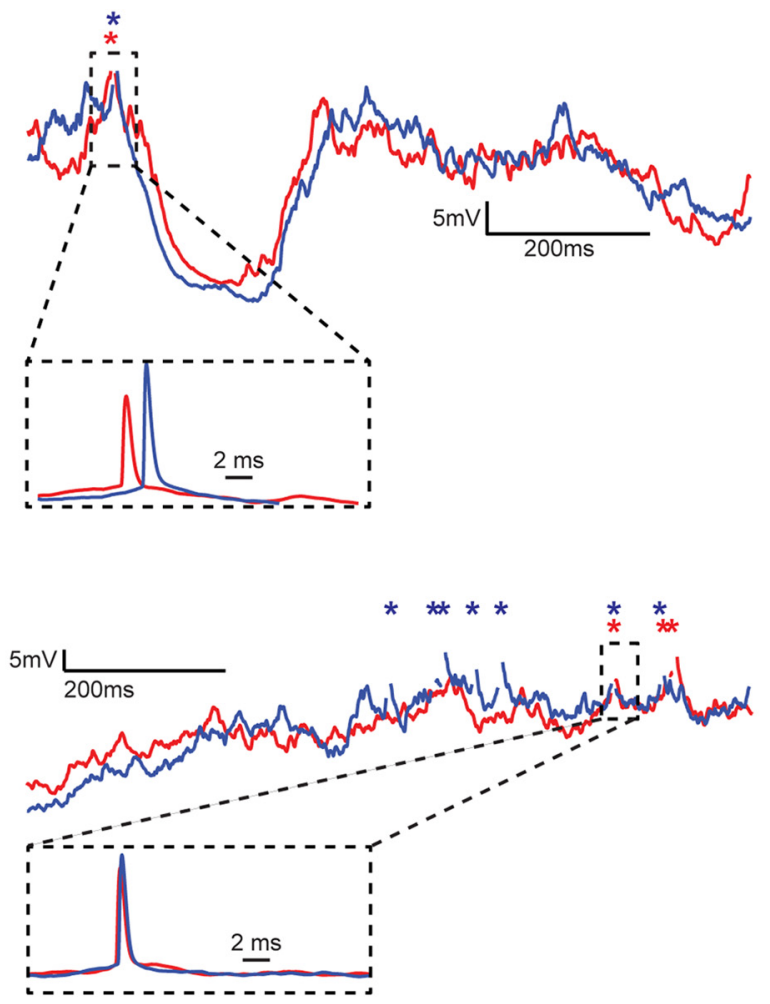

b

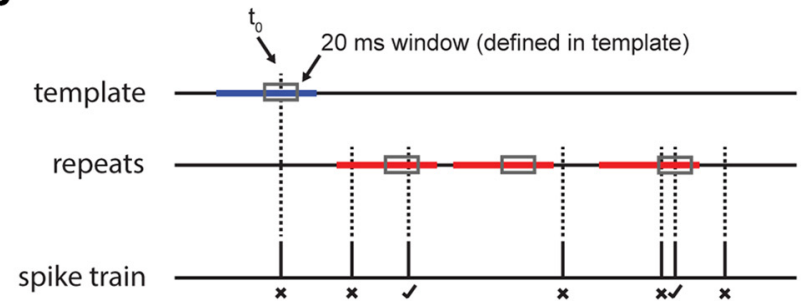

C

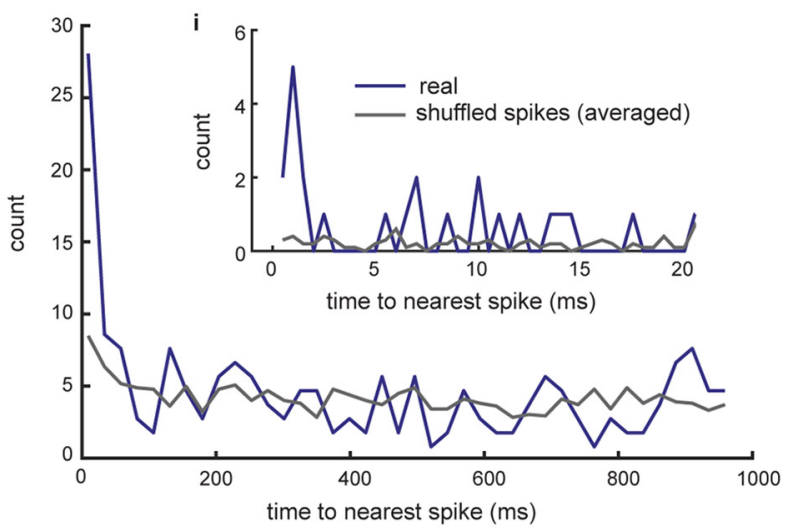

d

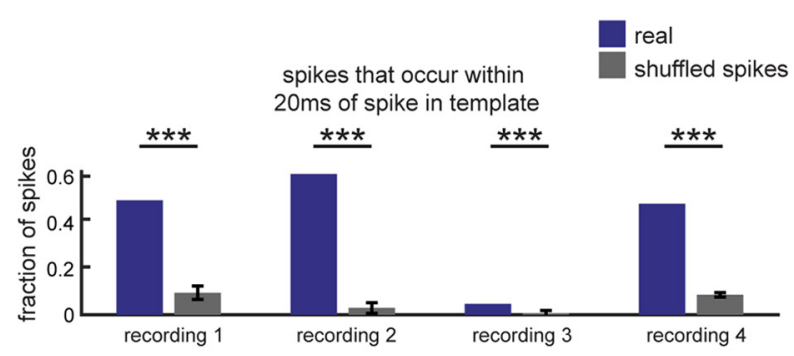

e
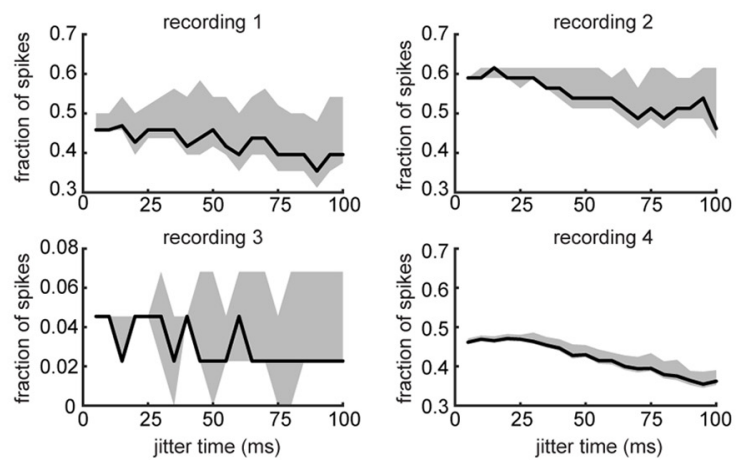

Figure 7. Precision of spikes within repeats. $\boldsymbol{a}$, Two examples (from recording 1) of spikes reoccurring with $10 \mathrm{~ms}$ precision ( $2.5 \mathrm{~ms}$ in top example, $0.5 \mathrm{~ms}$ in bottom example) within template-repeat pairs in spike-removed membrane voltage recordings. Spike locations are marked with *. Spikes are shown in the inset. $\boldsymbol{b}$, Method for quantifying spike temporal precision. For each spike within a template, a $20 \mathrm{~ms}$ window around the spike is defined. The $t_{0}$ point is the time index of the spike in the template. For every repeat with a spike, the time between $t_{0}$ and the nearest spike is recorded. Repeats containing spikes within $20 \mathrm{~ms}$ of $t_{0}$ are marked (check mark). Those spikes are considered precise. Each spike was counted only once (i.e., a marked spike could not be marked by another template). The fraction of spikes within $20 \mathrm{~ms}$ of the original is then calculated (in this diagram, 2 of $7=0.286$ ). c, Representative histogram for recording 1 (blue) showing the time between $t_{0}$ and the nearest spike in templates and their repeats (combined from 625 templates and 7173 repeats) truncated to 1000 and $20 \mathrm{~ms}$ (inset). Note that the time bin in the main panel $(20 \mathrm{~ms})$ is different from the time bin in the inset $(0.5 \mathrm{~ms})$. The same metric was calculated for the case when spikes were shuffled (gray line, average of 10 shuffles). $\boldsymbol{d}$, Fraction of spikes in repeats occurring within $20 \mathrm{~ms}$ of the spike in their templates for each recording (blue bars). Gray bars, Shuffled spikes. Recording 1: $t_{(9)}=27.2, p=5 \times 10^{-10}$, effect size $=1.79$; recording 2: $t_{(9)}=69.6$, $p=1 \times 10^{-13}$, effect size $=3.51$; recording 3: $t_{(9)}=9.79, p=5 \times 10^{-6}$, effect size $=0.34$; recording 4: $t_{(9)}=113, p=2 \times 10^{-15}$, effect size $=3.7\left(^{* * *} p<10^{-5}\right.$, Student's $t$ test; $n=$ 10 spike shuffles). Recordings were excluded if they had no spike-containing templates and repeats. Bar graphs show the mean \pm SD. $\boldsymbol{e}$, Fraction of precise spikes as a function of jitter time for the recordings analyzed in $\boldsymbol{d}$. Data are shown as the median (black) and the extent of the first/third quartile (gray).

spikes and repeats is not trivially explained by limitations of the repeat-finding algorithm.

Since spikes co-occurred with repeats, and repeats were found to have highly precise components, we asked whether spikes also occurred with high precision. Within template-repeat pairs in which the template contained a spike, spikes tended to occur within repeats with a surprising degree of temporal precision. That is, spikes in repeats tended to occur close to the position of spikes in their templates (Fig. 7a). To quantify the temporal precision, for each template-repeat pair where the template contained a spike we searched for a spike near each repeat, only counting each spike and repeat once to avoid double counting due to overlapping repeats (Fig. $7 b$ ). In a representative recording, we found a peak interval between spikes in the template and repeats between 0 and $20 \mathrm{~ms}$ (Fig. 7c). This peak disappears if the spikes within the recording are shuffled (Fig. $7 c$ ). In the four recordings with at least one spike-containing template and repeat, spikes were more likely to reoccur within $20 \mathrm{~ms}$ than in traces with shuffled spike times (Fig. $7 d$ ). The shuffled spike control ensures that any existing relationship between spike timing and repeat timing is eliminated. While this precision in spike timing was observed, we wondered whether it was correlated with the similarity between 
the template-repeat pair. To evaluate this, we computed the minimum time difference between spikes in templates and repeats and correlated that value to the template-repeat Pearson's $r$ value. For the four suitable recordings (recordings 1-4), no correlation was observed (recording $1: r=-0.04, p=0.59, n=175$; recording 2: $r=-0.076, p=0.25, n=235$; recording $3: r=$ $-0.41, p=0.3, n=8$; recording $4: r=-0.1, p=10^{-4}, n=$ 1392). In analyzing extracellular recordings, jitter analysis is often used to probe the temporal precision of neuronal processes (Hatsopoulos et al., 2003; Amarasingham et al., 2012). We used it to determine at what jitter interval spike precision was disrupted in spike-containing template-repeat pairs. In the four analyzed recordings, increased jitter time tended to reduce the fraction of precise spikes, as expected (Fig. 7e); however, the number of preshuffled precise spikes in recordings 1-3 was already low (2-23 spikes), much lower than in extracellular recording studies using this technique (Alvarado-Rojas et al., 2013; Diba et al., 2014). Because of this, an accurate estimate of the jitter time at which spike precision disappears could not be easily computed. Thus, while jitter analysis corroborated the existence of spike precision, it alone could not be used to quantify it.

\section{Discussion}

Repeated sequences of spiking activity in the hippocampus have previously been observed on the 100-300 ms timescale in the form of replay during SWRs (Nádasdy et al., 1999; Louie and Wilson, 2001; Dragoi and Tonegawa, 2011), but longer sequences have not been studied. Prior studies that have investigated long repeated patterns of intracellular activity were performed in vitro or in anesthetized preparations (Ikegaya et al., 2004, 2008; Mokeichev et al., 2007), leaving open the question of the existence and possible function of such long repeated patterns in the awake brain. In this study, we found 900-ms-long segments of repeated intracellular activity in CA1 neurons of awake, behaving mice, suggesting the presence of long presynaptic repeated sequences of spiking activity that occur independently of network state.

Using statistical controls, we determined that repeats occur more often in real recordings than in stochastically generated surrogate data. Moreover, the repeats tended to co-occur with spikes: $82 \%$ of spikes occurred during repeats. Finally, the repeated subthreshold activity and the spiking activity within repeats reoccurred with 10 and $20 \mathrm{~ms}$ precision, respectively. These results provide strong evidence that long patterns of activity are repeated and transmitted to downstream neurons, suggesting a longer timescale of information transmission than previously thought.

\section{Significance of long-timescale repeated activity}

The CA1 network can operate with exquisite temporal precision, and structured activity patterns have been observed at the $100-$ $300 \mathrm{~ms}$ timescale, which is associated with particular network states such as replay of sequences during SWRs (Nádasdy et al., 1999) and theta (Dragoi and Buzsáki, 2006) and gamma oscillations (Carr et al., 2012). In this study, we present evidence of repeated activity patterns that are both longer than previously described in CAl and independent of network state. Given that potentially thousands of cells are reactivated in a particular sequence during memory recall (Schmidt et al., 2012), it is plausible that the long repeated activity we observe may reflect such reactivation. We note that our findings represent a lower limit on the duration and prevalence of precisely repeated sequences of subthreshold activity, which might be observed if longer segments (e.g., hours or days, instead of minutes) of intracellular dynamics could be analyzed. Prior studies of reactivation may have been limited by the number of recorded cells and therefore the full duration of such repeated activity may not have been characterized. For example, long reactivation of sequences is difficult to detect using extracellular probes if spiking is spatially sparse. New technologies that can record orders of magnitude more cells may reveal longer timescales of reactivation from extracellular recordings.

The fact that repeats occur both within and outside of sharpwave ripples, which have been strongly implicated in spatial memory, suggests that the brain has multiple mechanisms for generating the same activity pattern multiple times. Since repeats occur both during and outside of canonical LFP oscillations such as theta and gamma, it would seem that their genesis is independent of the mechanisms of network rhythmogenesis. Repeats and sharp-wave ripple replay may carry the same type of information, perhaps with different functions (also suggested by the fact that during replay previous activity is compressed in time), or repeats may carry complementary information (e.g., about the internal state of the animal). Since the same repeats are observed in different network states, it is possible that the same information might be used differently in the brain at different times (e.g., for navigation vs memory formation vs planning), perhaps depending on the ongoing state of the network.

\section{Evidence for long-timescale information transmission}

To date, little attention has been focused on how sequences of spiking activity are transmitted to single neurons downstream. Sequences of activity encoding information over time could be transmitted in multiple ways. On one extreme, a sequence of activity could propagate from one neural population to another in a one-to-one manner such that any single neuron receives information only about a single moment in the sequence. At the other extreme, postsynaptic cells could receive inputs from many single neurons that are active throughout the sequence such that the cell receives inputs across the entire sequence. Our discovery of long repeated subthreshold patterns of activity that likely reflect repeated presynaptic inputs to the neuron being recorded suggests single neurons receive long sequences of activity instead of only short parts of sequences.

The observed link between repeats and spikes, and the precision of repeated subthreshold and spiking activity suggest that this repeated activity could be important for information transmission in the network. Three findings support this idea. First, the precise reoccurrence of subthreshold patterns above chance levels suggests that this activity is driven by repeated patterns of spiking activity that provide inputs to the recorded cell. These repeated presynaptic spiking patterns may not be identical but are similar enough to produce very similar postsynaptic activity, in conjunction with the passive biophysical properties and active conductances of recorded neurons. High-resolution recordings with calcium or voltage sensors from both dendrites and soma of CA1 cells might help to dissect the roles of inputs and intrinsic mechanisms in precisely reproducing repeated patterns of postsynaptic activity. Repeated patterns of spiking activity in the inputs to CA1 neurons could arise in CA3. Spiking sequences in CA3 pyramidal cells are thought to stem from recurrent connections between CA3 pyramidal cells (Levy, 1996; Lisman, 1999). Alternately, these repeated patterns could arise from a combination of inputs from entorhinal cortex, CA3, and local interneurons. While with our data it is not possible to establish exactly where the repeated inputs to the CA1 neuron recorded come from, the prevalence of repeats and their strong correlation with 
spiking would suggest that the two main inputs to CA1, CA3, and/or entorhinal cortex play an important role. Regardless of the exact regions involved in providing the repeated input patterns, it is possible that the activity represents network attractor states, onto which the network converges, even starting from slightly different initial conditions. In a pathological context, it is possible that the epileptic state shares some features with, and may evolve from, such persistent network attractor states.

Second, spikes occur preferentially during repeats, suggesting that repeated activity is more likely to drive neuronal output than nonrepeating activity. This could arise because the patterns of network activity that are more likely to drive repeats are also more likely to drive spiking. Indeed, these repeated subthreshold patterns may reflect periods of highly coordinated activity in the inputs of a cell that are more likely to make the cell fire. For example, large depolarizations that drive spiking in the template are also likely to drive spiking during repeats, contingent on the state of the neuron before the beginning of the repeat and on variability in the ongoing synaptic inputs. It is important to note that while most spikes occurred during repeated subthreshold activity, not all repeats included a spike. Therefore, we cannot conclude that repeats, at a broad level, drive spiking activity.

Third, we observed high levels of precision in both the membrane potential repeats and in spike patterns. In the membrane potential, we found that, although the majority of the correlation between templates and repeats is created by low-frequency components, the correlation between templates and repeats in high frequencies $(\geq 100 \mathrm{~Hz})$ is greater than would be expected by chance, suggesting a level of precision on the $10 \mathrm{~ms}$ timescale. In spike patterns, we found that templates with spikes had repeats with spikes within $20 \mathrm{~ms}$ of the template spike at a rate above what would be expected by chance. We have observed no evidence of significant repeat or spike precision on the single-millisecond timescale. Nevertheless, the precision we did find is consistent with the observed spike precision reported previously (Mainen and Sejnowski, 1995). For example, the spike jitter in CA1 pyramidal cells in response to EPSPs was found to be in the $10-20 \mathrm{~ms}$ range (Mainen and Sejnowski, 1995; Fricker and Miles, 2000). Thus, the high precision of spikes in repeats provides further support that these repeated patterns may be due to repeated structured activity in the inputs of recorded cells.

\section{Comparison to results from anesthetized cortex}

The structure of subthreshold activity differs in important ways between awake and anesthetized brain states, which could have significant effects on how repeated activity is detected and interpreted. During anesthesia, periodic up-down states are often observed intracellularly, which changes the structure of the recording and likely influences information processing (Bermudez Contreras et al., 2013). On the other hand, up-down states are rare in in the hippocampus and in awake animals (with exceptions; Engel et al., 2016). Mokeichev et al. (2007) report slight to significant bimodality in the membrane potential of their recordings, an expected effect of anesthesia, while our recordings show no consistent bimodality. The lack of this bimodal structure to our recordings likely contributed to $\sim 1000$-fold fewer repeats being found in our preparation. When we ran our repeat-finding algorithm on recordings from anesthetized cortex with clear updown states, we found many more repeats (e.g., $\sim 25,000$ for a 4 min recording), as expected. Thus, we conclude that repeats are more likely to be found in recordings with bimodal membrane voltage distributions. There are two other differences in our repeat-finding approaches from Mokeichev et al. (2007) that may also contribute to a difference in the number of detected repeats. First, our recordings were all shorter than the recording duration used in the study by Mokeichev et al. (2007) (10-20 min), so we had fewer candidate templates to use to search for repeats. Second, there were differences in the way we searched for repeats. Mokeichev et al. (2007) used a tiered search at two timescales, producing a similarity score of two segments at a low resolution [ $1 \mathrm{~s}$, termed the low-resolution index (LRI)] and at high resolution [100 ms; termed the high-resolution index (HRI)]. Recording segments passing a low-resolution threshold of correlation coefficient $r=0.45$ were considered to be repeats. We used only one threshold set to $r=0.80$, which predictably led to fewer repeats being found in our recordings. We elected to not use the LRI-HRI approach in our study to maintain simplicity in reporting results as correlation coefficients rather than abstract scores. A more recent approach, based on detecting postsynaptic potentials (Ikegaya et al., 2008) could not be used because we could not reliably detect individual PSPs, likely due to the structure of membrane potential fluctuations in awake animals, which receive large barrages of inputs from presynaptic neurons.

\section{Statistical controls to establish significance}

The significance of spontaneously occurring intracellular repeats has been controversial due to the difficulty in creating proper statistical methods of controlling for correlated segments that emerge randomly. We used rigorous statistical controls (Mokeichev et al., 2007) to establish that even though spontaneous activity in the awake brain is thought to be highly desynchronized, there are still cells with precisely repeated subthreshold patterns over long timescales in hippocampal CA1. The three surrogate generation strategies shown in that study represented a significant advance in analyzing repeated intracellular activity; however, we found that they needed two modifications to make them suitable for analyzing recordings from awake, behaving animals. First, we replaced the Poisson simulation from the study by Mokeichev et al. (2007), which produced surrogate data that were not similar to our recordings, with a stochastic synaptic input model (Paninski et al., 2012), which better reproduced membrane dynamics of awake recordings such as unimodality, frequent, nonseparable synaptic inputs, and baseline variation. Second, for interval shuffling, we decreased the duration of the longest possible contiguous segment from 450 to $100 \mathrm{~ms}$. This decreased the effect of baseline variation on the interval-shuffling procedure. The 450 ms threshold was appropriate for breaking up and rearranging bimodal membrane potential traces like up and down states in anesthetized preparations but insufficiently shuffled our awake recordings, as described in the "Establishing significance of repeated patterns using statistical controls" section. While surrogate generation strategies cannot definitively prove the nonstochastic emergence of repeats, they are a reliable starting point for these analyses. Beyond these statistical controls, we also established the significance of these repeated patterns by characterizing their precision and relationship to spike output.

In summary, we find unexpectedly long repeated subthreshold patterns of activity in CA1 of awake, behaving mice, which occur independently of network state and are strongly correlated with spiking output. These results suggest that long sequences of activity are transmitted to single postsynaptic neurons. These findings help constrain possible models of information encoding and transmission in the awake brain.

\section{References}

Abeles M (1991) Corticonics: neural circuits of the cerebral cortex. Cambridge, UK: Cambridge UP. 
Alvarado-Rojas C, Lehongre K, Bagdasaryan J, Bragin A, Staba R, Engel J Jr, Navarro V, Le Van Quyen M (2013) Single-unit activities during epileptic discharges in the human hippocampal formation. Front Comput Neurosci 7:140. CrossRef Medline

Amarasingham A, Harrison MT, Hatsopoulos NG, Geman S (2012) Conditional modeling and the jitter method of spike resampling. J Neurophysiol 107:517-531. CrossRef Medline

Bennett C, Arroyo S, Hestrin S (2013) Subthreshold mechanisms underlying state-dependent modulation of visual responses. Neuron 80:350-357. CrossRef Medline

Bermudez Contreras EJ, Schjetnan AG, Muhammad A, Bartho P, McNaughton BL, Kolb B, Gruber AJ, Luczak A (2013) Formation and reverberation of sequential neural activity patterns evoked by sensory stimulation are enhanced during cortical desynchronization. Neuron 79: 555-566. CrossRef Medline

Carr MF, Jadhav SP, Frank LM (2011) Hippocampal replay in the awake state: a potential substrate for memory consolidation and retrieval. Nat Neurosci 14:147-153. CrossRef Medline

Carr MF, Karlsson MP, Frank LM (2012) Transient slow gamma synchrony underlies hippocampal memory replay. Neuron 75:700-713. CrossRef Medline

Carrillo-Reid L, Yang W, Bando Y, Peterka DS, Yuste R (2016) Imprinting and recalling cortical ensembles. Science 353:691-694. CrossRef Medline

Constantinople CM, Bruno RM (2011) Effects and mechanisms of wakefulness on local cortical networks. Neuron 69:1061-1068. CrossRef Medline

Contreras D, Steriade M (1995) Cellular basis of EEG slow rhythms: a study of dynamic corticothalamic relationships. J Neurosci 15:604-622. Medline

Davidson TJ, Kloosterman F, Wilson MA (2009) Hippocampal replay of extended experience. Neuron 63:497-507. CrossRef Medline

Diba K, Amarasingham A, Mizuseki K, Buzsáki G (2014) Millisecond timescale synchrony among hippocampal neurons. J Neurosci 34:1498414994. CrossRef Medline

Diekelmann S, Born J (2010) The memory function of sleep. Nat Rev Neurosci 11:114-126. CrossRef Medline

Diesmann M, Gewaltig MO, Aertsen A (1999) Stable propagation of synchronous spiking in cortical neural networks. Nature 402:529-533. CrossRef Medline

Dragoi G, Buzsáki G (2006) Temporal encoding of place sequences by hippocampal cell assemblies. Neuron 50:145-157. CrossRef Medline

Dragoi G, Tonegawa S (2011) Preplay of future place cell sequences by hippocampal cellular assemblies. Nature 469:397-401. CrossRef Medline

Engel TA, Steinmetz NA, Gieselmann MA, Thiele A, Moore T, Boahen K (2016) Selective modulation of cortical state during spatial attention. Science 354:1140-1144. CrossRef Medline

Fiete IR, Senn W, Wang CZ, Hahnloser RH (2010) Spike-time-dependent plasticity and heterosynaptic competition organize networks to produce long scale-free sequences of neural activity. Neuron 65:563-576. CrossRef Medline

Fricker D, Miles R (2000) EPSP amplification and the precision of spike timing in hippocampal neurons. Neuron 28:559-569. CrossRef Medline

Girardeau G, Benchenane K, Wiener SI, Buzsáki G, Zugaro MB (2009) Selective suppression of hippocampal ripples impairs spatial memory. Nat Neurosci 12:1222-1223. CrossRef Medline

Haider B, Duque A, Hasenstaub AR, McCormick DA (2006) Neocortical network activity in vivo is generated through a dynamic balance of excitation and inhibition. J Neurosci 26:4535-4545. CrossRef Medline

Harvey CD, Collman F, Dombeck DA, Tank DW (2009) Intracellular dynamics of hippocampal place cells during virtual navigation. Nature 461: 941-946. CrossRef Medline

Harvey CD, Coen P, Tank DW (2012) Choice-specific sequences in parietal cortex during a virtual-navigation decision task. Nature 484:62-68. CrossRef Medline

Hatsopoulos N, Geman S, Amarasingham A, Bienenstock E (2003) At what time scale does the nervous system operate? Neurocomputing 52:25-29.

Ikegaya Y, Aaron G, Cossart R, Aronov D, Lampl I, Ferster D, Yuste R (2004) Synfire chains and cortical songs: temporal modules of cortical activity. Science 304:559-564. CrossRef Medline

Ikegaya Y, Matsumoto W, Chiou HY, Yuste R, Aaron G (2008) Statistical significance of precisely repeated intracellular synaptic patterns. PLoS One 3:e3983. CrossRef Medline

Jadhav SP, Kemere C, German PW, Frank LM (2012) Awake hippocampal sharp-wave ripples support spatial memory. Science 336:1454-1458. CrossRef Medline

Kodandaramaiah SB, Franzesi GT, Chow BY, Boyden ES, Forest CR (2012) Automated whole-cell patch-clamp electrophysiology of neurons in vivo. Nat Methods 9:585-587. CrossRef Medline

Levy WB (1996) A sequence predicting CA3 is a flexible associator that learns and uses context to solve hippocampal-like tasks. Hippocampus 6:579-590. CrossRef Medline

Li CY, Poo MM, Dan Y (2009) Burst spiking of a single cortical neuron modifies global brain state. Science 324:643-646. CrossRef Medline

Lisman JE (1999) Relating hippocampal circuitry to function: recall of memory sequences by reciprocal dentate-CA3 interactions. Neuron 22 : 233-242. CrossRef Medline

Louie K, Wilson MA (2001) Temporally structured replay of awake hippocampal ensemble activity during rapid eye movement sleep. Neuron 29:145-156. CrossRef Medline

Luczak A, Maclean JN (2012) Default activity patterns at the neocortical microcircuit level. Front Integr Neurosci 6:30. CrossRef Medline

Luczak A, Barthó P, Harris KD (2009) Spontaneous events outline the realm of possible sensory responses in neocortical populations. Neuron 62:413425. CrossRef Medline

MacLean JN, Watson BO, Aaron GB, Yuste R (2005) Internal dynamics determine the cortical response to thalamic stimulation. Neuron 48:811823. CrossRef Medline

Mainen ZF, Sejnowski TJ (1995) Reliability of spike timing in neocortical neurons. Science 268:1503-1506. CrossRef Medline

Mauk MD, Buonomano DV (2004) The neural basis of temporal processing. Annu Rev Neurosci 27:307-340. CrossRef Medline

Miller JE, Ayzenshtat I, Carrillo-Reid L, Yuste R (2014) Visual stimuli recruit intrinsically generated cortical ensembles. Proc Natl Acad Sci U S A 111:E4053-E4061. CrossRef Medline

Mokeichev A, Okun M, Barak O, Katz Y, Ben-Shahar O, Lampl I (2007) Stochastic emergence of repeating cortical motifs in spontaneous membrane potential fluctuations in vivo. Neuron 53:413-425. CrossRef Medline

Nádasdy Z, Hirase H, Czurkó A, Csicsvari J, Buzsáki G (1999) Replay and time compression of recurring spike sequences in the hippocampus. J Neurosci 19:9497-9507. Medline

Oram MW, Wiener MC, Lestienne R, Richmond BJ (1999) Stochastic nature of precisely timed spike patterns in visual system neuronal responses. J Neurophysiol 81:3021-3033. CrossRef Medline

Paninski L, Vidne M, DePasquale B, Ferreira DG (2012) Inferring synaptic inputs given a noisy voltage trace via sequential Monte Carlo methods. J Comput Neurosci 33:1-19. CrossRef Medline

Rolston JD, Wagenaar DA, Potter SM (2007) Precisely timed spatiotemporal patterns of neural activity in dissociated cortical cultures. Neuroscience 148:294-303. CrossRef Medline

Roxin A, Hakim V, Brunel N (2008) The statistics of repeating patterns of cortical activity can be reproduced by a model network of stochastic binary neurons. J Neurosci 28:10734-10745. CrossRef Medline

Schmidt B, Marrone DF, Markus EJ (2012) Disambiguating the similar: the dentate gyrus and pattern separation. Behav Brain Res 226:56-65. CrossRef Medline

Shmiel T, Drori R, Shmiel O, Ben-Shaul Y, Nadasdy Z, Shemesh M, Teicher M, Abeles M (2005) Neurons of the cerebral cortex exhibit precise interspike timing in correspondence to behavior. Proc Natl Acad Sci U S A 102:18655-18657. CrossRef Medline

Singer AC, Talei Franzesi GT, Kodandaramaiah SB, Flores FJ, Cohen JD, Lee AK, Borgers C, Forest CR, Kopell NJ, Boyden ES (2017) Mesoscaleduration activated states gate spiking in response to fast rises in membrane voltage in the awake brain. J Neurophysiol 118:1270-1291. CrossRef Medline

Steriade M, Timofeev I, Grenier F (2001) Natural waking and sleep states: a view from inside neocortical neurons. J Neurophysiol 85:1969-1985. CrossRef Medline

Stern EA, Kincaid AE, Wilson CJ (1997) Spontaneous subthreshold membrane potential fluctuations and action potential variability of rat corticostriatal and striatal neurons in vivo. J Neurophysiol 77:1697-1715. CrossRef Medline

Welford BP (1962) Note on a method for calculating corrected sums of squares and products. Technometrics 4:419-420. CrossRef

Yuhas RHG (1992) Discrimination among semiarid landscape endmembers using the Spectral Angle Mapper (SAM) algorithm. Washington, DC: NASA 19940012238. 